\title{
On the attainable set for a class of triangular systems of conservation laws
}

\author{
Boris Andreianov*, Carlotta Donadello ${ }^{\dagger}$ \\ Shyam S. Ghoshal ${ }^{\ddagger}$ and Ulrich Razafison ${ }^{\S}$
}

March 29, 2014

\begin{abstract}
We explore attainability for a special class of triangular systems of conservation laws, not necessarily strictly hyperbolic, which includes the system of multicomponent chromatography. Roughly speaking, such systems consist of linear continuity equations coupled with a scalar genuinely nonlinear conservation law. The classical Keyfitz-Kranzer system is also included, with minor modifications. We prove that the backward solutions we construct are appropriate solutions of the system in view of the classical theories for general conservation laws. In particular, we get isentropic solutions whenever nontrivial entropies for the system are defined. We give numerical examples of the isentropic backward resolution of such systems for attainable target data.
\end{abstract}

* Laboratoire de Mathematiques de Besançon CNRS UMR 6623, Université de Franche-Comté, 16 route de Gray 25030 Besançon, France and Institut für Mathematik, Technische Universität Berlin, 136 Straße des 17. Juni, Berlin, Germany. E-mail: boris.andreianov@univ-fcomte.fr

${ }^{\dagger}$ Laboratoire de Mathematiques de Besançon CNRS UMR 6623, Université de Franche-Comté, 16 route de Gray 25030 Besançon, France. E-mail: carlotta.donadello@univ-fcomte.fr

${ }^{\ddagger}$ Laboratoire de Mathematiques de Besançon CNRS UMR 6623, Université de Franche-Comté, 16 route de Gray 25030 Besançon, France and Institut de Mathématiques de Toulouse, Université Paul Sabatier, 118, route de Narbonne, 31062 Toulouse Cedex 9. E-mail: s.s.ghoshal@gmail.com

${ }^{\S}$ Laboratoire de Mathematiques de Besançon CNRS UMR 6623, Université de Franche-Comté, 16 route de Gray 25030 Besançon, France. E-mail: ulrich.razafison@univ-fcomte.fr 


\section{Contents}

1 Introduction $\quad 3$

1.1 Statement of the problem, related literature . . . . . . . . . . . 3

1.2 Main ideas and structure of the paper . . . . . . . . . . . . 4

2 Attainability revisited $\quad 6$

2.1 Characterization of the set $\mathcal{A}_{T} \ldots \ldots \ldots \ldots$

2.2 On the structure of the set $\mathcal{A}_{T} \ldots \ldots \ldots \ldots$

3 Systems endowed with entropies 9

3.1 Entropies for a system in the form $(14) \ldots \ldots . \ldots 9$

3.2 Strong generalized solutions for continuity equations . . . . . . . . . 11

3.3 Main result on non-resonant systems . . . . . . . . . . . . . . . . 13

3.4 On the Keyfitz-Kranzer system . . . . . . . . . . . . . . . . . . . . . 14

4 Resonant systems $\quad \mathbf{1 7}$

4.1 Attainability and approximate attainability result . . . . . . . . . 17

4.2 On the relation between results of Theorems 3.1 and $4.1 \quad \ldots . . . \quad 18$

4.3 Examples of forward and backward non-uniqueness for the resonant case 19

5 Numerics $\quad 20$

5.1 The numerical method . . . . . . . . . . . . . . . . . 20

5.2 Numerical results . . . . . . . . . . . . . . . . . . . . 22

6 Conclusions $\quad 24$

7 Appendix: backward construction for (6) revisited $\mathbf{2 5}$ 7.1 The isentropic backward algorithm on a bounded domain . . . . . . 26

7.2 Target state given on an unbounded domain . . . . . . . . . . . 29

$\begin{array}{ll}\text { References } & 30\end{array}$ 


\section{Introduction}

\subsection{Statement of the problem, related literature}

In this paper we consider triangular systems of conservation laws of the form

$$
\left\{\begin{array}{l}
u_{t}+f(u)_{x}=0, \\
\left(v_{i}\right)_{t}+\left(g_{i}(u) v_{i}\right)_{x}=0, \quad \text { for } i=1, \ldots, m
\end{array}\right.
$$

where $f$ and $g_{i}$, for $i=1, \ldots, m$, are functions of class $C^{1}(\mathcal{I})$ and $f$ is a strictly convex function. Here $\mathcal{I}$ can be a sub-interval of $\mathbb{R}$, but we will assume $\mathcal{I}=\mathbb{R}$ to shorten the presentation.

Our aim is to characterize the set $\mathfrak{A}_{T}$ of profiles $U_{T}=\left(u_{T}, v_{1, T}, \ldots, v_{m, T}\right) \in$ $L^{\infty}\left(\mathbb{R} ; \mathbb{R}^{m+1}\right)$ that a solution of $(1)$ can attain at a fixed time $T>0$ starting from a suitable initial condition. In view of the applications, we are particularly interested in the explicit construction of a backward solver.

First of all, let us recall what is known when $m=0$. To our knowledge, the problem of finding states which the solution of a scalar conservation laws with convex flux

$$
u_{t}+f(u)_{x}=0,
$$

can attain by an initial or boundary data control has been encountered in $[7,25,1]$.

In [7], Ancona and Marson indagate exact controllability for the initial boundary value problem. The authors use the theory of generalized characteristics introduced by Dafermos in [13], in the case of general strictly convex flux and describe, for any given time $t>0$, the set of states which are attainable in time $t$ starting from the initial condition $u_{0}=0$ by a control on the boundary data alone.

The approximate controllability problem for the Burgers equation has been considered by Horsin in [25], by using the return method introduced by Coron, [11].

The recent works by Adimurthi, Ghoshal and Gowda [1, 2] (see also [20]) focus on the exact controllability problem for the equation (2) in the setting of strictly convex, superlinearly growing flux. The authors treat the initial value problem and the boundary value problem in the half-space and in a strip with two boundaries. By using the Lax-Oleinik formula, they give an elegant algorithm which constructs explicitly the desired initial data control, and they give a concise characterization of the attainable set which we exploit in the present work, see Proposition 2.1.

Finally, let us mention that for the viscous Burgers equation, constant states reachable by boundary controls has been studied using the Cole-Hopf transformation by Glass and Guerrero [21], Guerrero and Imanuvilov [24]. More recently, Léautaud used the vanishing viscosity approach to study the uniform controllability of scalar conservation laws in [28].

Due to the irreversibility of hyperbolic systems, construction of physically relevant backward solvers is a very delicate problem and it is widely open in general situations. In the present work, we exploit the special form of (1) to prove existence of backward solutions starting from any profile $U_{T}$ in the attainable set $\mathfrak{A}_{T}$. Our results apply to some well-known examples of systems of conservation laws (the Keyfitz-Kranzer system; the multi-component chromatography system).

Roughly speaking, the structure of (1) (and of the Keyfitz-Kranzer system, treated analogously) combines the two frameworks in which a backward construction is available: convex scalar conservation laws and linear continuity equations with 
sufficiently regular coefficients. However, in order to apply the existing theories to our problem we have to face two difficulties. First, a backward solution for the scalar conservation law (the first line of (1)) may contain shock discontinuities. Therefore, the coefficients of the continuity equations (the second line of (1)) may be not regular enough to ensure existence of a backward solution, see [12] for some discussion in this direction. Second, even when a backward construction can be applied for each of the scalar equations in system (1), it is not clear a priori that the vector function $U=\left(u, v_{1}, \ldots, v_{m}\right)$ constructed component-wise is an admissible solution for the system (1).

We recall that weak solutions of systems of conservation laws are not unique in general. The literature contains different criteria to select physically relevant solutions and in particular, whenever a hyperbolic system is endowed with a physical entropy, solutions are judged physically relevant if and only if they dissipate entropy as time advances, see e.g. [33, 32, 14, 10]. An easy consequence of this definition is that admissible reversible solutions must be isentropic, i.e. they should preserve entropy. In the scalar case, isentropic solutions are characterized in [27]; continuity is their essential property. Isentropic solutions will play a key role in order to overcome the two aforementioned difficulties.

\subsection{Main ideas and structure of the paper}

Fine description of the attainability property for convex scalar conservation law is the first essential ingredient of our result for systems (1). Therefore we start by presenting an advantageous version of the backward resolution procedure for scalar conservation laws $u_{t}+f(u)_{x}=0$ with convex flux $f$ introduced by Adimurthi, Ghoshal and Veerappa Gowda in [1] (this version appeared as a remark in [20]). In particular, we give a simple detailed proof of the fact that for every target datum $u_{T}$ in the set $\mathcal{A}_{T}$ of states which are attainable at time $T$, there exists an isentropic solution (which is, therefore, both forward and backward entropy solution) on $[0, T]$. We refer to Ancona and Marson [7] for the first proof of this curious fact which follows from the theory of generalized characteristics of Dafermos (see, e.g., $[13,14]$ ).

To be specific, we prove that one can define on $\mathcal{A}_{T}$ an $L^{\infty}$-stable and $B V$-stable, $L^{1}$-contractive backward solver $S_{T}^{\leftarrow}:[0, T] \times \mathcal{A}_{T} \mapsto L^{\infty}(\mathbb{R})$ such that the function $u(t, \cdot):=S_{T}^{\leftarrow}(t) u_{T}$ is an isentropic solution of

$$
\left\{\begin{array}{l}
u_{t}+f(u)_{x}=0 \\
u(T, \cdot)=u_{T}
\end{array}\right.
$$

on the strip $[0, T] \times \mathbb{R}$. We construct explicitly this solution as the limit of a sequence of exact solutions $u_{n}$ to $u_{t}+f(u)_{x}=0$ such that each $u_{n}$ consists of alternating rarefaction and compression waves combined in such a way that singularities may appear only at the initial time $t=0$ and at the final time $t=T$, while the solution is regular on $[\delta, T-\delta] \times \mathbb{R}$, for every $\delta>0$.

Let us stress that the fact that the solution is isentropic opens the way to its numerical approximation, since it follows readily from uniqueness of entropy solutions for the auxiliary forward Cauchy problem

$$
\left\{\begin{array}{l}
w_{t}+(-f(w))_{x}=0 \\
\left.w\right|_{t=0}=u_{T}
\end{array}\right.
$$


that $S_{T}^{\leftarrow}(t) u_{T}=w(T-t)$. Therefore the backward isentropic solution can be approximated, e.g., by a standard monotone consistent finite volume numerical scheme for (3). The numerical simulations of this paper are based on this approach (see Section $5)$.

Turning to the analysis of system (1), we consider first the situation in which the system is non-resonant in the range of $u$ (a more precise statement will be given in Section 3). Note that the non-resonance condition can be checked a priori since $u$ satisfies the maximum principle. In this framework we are able to completely describe the entropies of the system. The entropies permit to recast the continuity equations contained in (1) into the form

$$
\begin{aligned}
& (A(t, x) v)_{t}+(B(t, x) v)_{x}=0 \\
& \text { with divergence-free field }(t, x) \mapsto(A(t, x), B(t, x))
\end{aligned}
$$

and $A \geq$ const $>0$. This reduction allows us to exploit another essential technical ingredient that is the theory of Panov [31] for equations of the special form (4). In the setting of generalized solutions of Panov, we get existence and uniqueness for the continuity equations, see [31]. Notice that Panov's generalized solutions are, in particular, time-reversible and they satisfy a renormalization property that is essential for the application of our results to the Keyfitz-Kranzer system (cf. [30]). Let us also mention the works of De Lellis et al. on the related theory of nearly incompressible vector fields which applies in the multi-dimensional setting (see [15] for a self-contained presentation).

Results on the case of systems endowed with nontrivial entropies, called "nonresonant", are presented in Section 3. Our result in the setting of non-resonant systems is optimal in the sense that the attainable set is fully described: one has $\mathfrak{A}_{T}=\mathcal{A}_{T} \times L^{\infty}\left(\mathbb{R} ; \mathbb{R}^{m}\right)$. For any given final state $U_{T} \in \mathcal{A}_{T} \times L^{\infty}\left(\mathbb{R} ; \mathbb{R}^{m}\right)$, there exists a unique bounded isentropic weak solution for the system $U$. Attainability for the Keyfitz-Kranzer system is also treated in Section 3, with the same tools. Let us mention in passing that we obtain a forward well-posedness theory for nonresonant case of (1) which is analogous to the theory of strong entropy solutions for the Keyfitz-Kranzer system (see $[18,30]$ ) but which is restricted to data that give rise to isentropic solutions (see Remarks 3.3,3.4).

In order to deal with the resonant case we develop a second approach, which we present in details in Section 4. Roughly speaking, we introduce the set $\mathbb{A}_{T}$, dense in $\mathcal{A}_{T}$ with respect to the $L^{1}$-norm, and for $u_{T} \in \mathbb{A}_{T}$ and under the local uniform convexity assumption

$$
\text { for all compact } K \subset I \text { there exists } \alpha(K)>0 \text { s. t. }\left.f^{\prime \prime}\right|_{K} \geq \alpha(K) \text {, }
$$

we find that $u(t, x)=S_{T}^{\leftarrow}(t) u_{T}(x)$ belongs to $W^{1, \infty}([0, T] \times \mathbb{R})$. This regularity is enough to apply the theory of renormalized solutions of continuity equations with mildly regular coefficients (see the classical paper by DiPerna-Lions, [16]; it should be stressed that renormalized solutions are time-reversible) to solve backwards the continuity equation. By analogy with the theory of Keyfitz-Kranzer system, we fix a notion of solution by considering that the function $U=\left(u, v_{1}, \ldots, v_{m}\right)$ is an admissible solution for the system if $u$ is an entropy solution for the scalar conservation law and the functions $v_{i}$ are renormalized solutions of the companion continuity equations in the sense of DiPerna and Lions. Our result in this framework can be read as follows : we obtain exact controllability of our system in finite time $T$ in the 
set $\mathbb{A}_{T} \times L^{\infty}\left(\mathbb{R} ; \mathbb{R}^{m}\right)$ and then, by density, we deduce approximate controllability in the set $\mathfrak{A}_{T}$.

Let us stress that, under the non-resonance condition and the above regularity restrictions on $f$ and $u_{T}$, solutions obtained by the method of Section 4 and by the one of Section 3 coincide as shown by Panov in [31, Sect. 7].

The last part of this paper is devoted to numerical simulations for the problem obtained by combining a classical monotone finite volume scheme for (3) with upwind type scheme used by Gosse and James, [23] (see also [17]), for the continuity equation with one-side Lipschitz coefficients. Numerical examples are given for computation of $U_{0}$ from $U_{T}$. We also illustrate the results of this paper by comparing $U_{T}$ to the state obtained at $t=T$ by forward resolution of (1) starting from the so constructed initial control $U_{0}$.

The structure of the paper is the following. In Section 2 we present a slight modification of the construction in [1, 2]; a detailed justification is postponed to Appendix. In Section 3 we discuss the structure of the entropies of a system of the form (14), we briefly recall some results on generalized solutions, see [31, 15], and we prove the exact controllability of all states $U_{T} \in \mathcal{A}_{T} \times\left(L^{\infty}(\mathbb{R})\right)^{m}$ under the assumption that the system (1) admits nontrivial entropies. Analogous results are obtained for the Keyfitz-Kranzer system. Then in Section 4 we prove exact controllability of all states $U_{T} \in \mathbb{A}_{T} \times\left(L^{\infty}(\mathbb{R})\right)^{m}$ under the assumption (5) by using classical results on continuity equations with Sobolev coefficients, see [16]. In Section 5 we discuss a numerical algorithm for backward resolution of (1) and give numerical examples.

\section{Attainability revisited}

The goal of this section is to recall the characterization of the set $\mathcal{A}_{T}$ of states attainable at time $T$ by solutions of a convex conservation law, to point out simple relations between $\mathcal{A}_{T}$ and $\mathcal{A}_{T+\delta}, \delta>0$, and to stress the fact that every attainable state is attainable by a (unique) isentropic solution.

\subsection{Characterization of the set $\mathcal{A}_{T}$}

Let us recall the following fundamental result that can be inferred from [7] and [1, 2], and provide a simple and constructive proof of it.

Proposition 2.1. Consider a scalar conservation law

$$
u_{t}+f(u)_{x}=0,
$$

with convex flux $f$ of class $C^{1}$.

Let $T>0$, and consider the whole of $\mathbb{R}$ as the spatial domain. Then

(i) The set of states in $L^{\infty}(\mathbb{R})$ attainable by Kruzhkov entropy solutions of (6) at time $T$ is given by

$$
\begin{aligned}
\mathcal{A}_{T}(\mathbb{R}, f)= & \left\{u \in L^{\infty}(\mathbb{R}): \exists \rho: \mathbb{R} \rightarrow \mathbb{R},\right. \text { right continuous, } \\
& \text { nondecreasing such that } \left.f^{\prime}(u)=\frac{x-\rho(x)}{T}\right\} .
\end{aligned}
$$


(ii) Furthermore, for every $u_{T} \in \mathcal{A}_{T}(\mathbb{R}, f)$ there exists a unique isentropic solution $u$ of $(6)$ on $[0, T] \times \mathbb{R}$ that verifies $u(T, \cdot)=u_{T}$.

The explicit and very practical representation (7) has been stated in [1, 2, 20]. Notice that the statement Proposition 2.1(ii), which is not highlighted in the relevant literature, is in fact essential for the applications we have in mind. The result of Proposition 2.1 follows from the theory of generalized characteristics of Dafermos $[13,14]$ (see in particular the arguments developed by Ancona and Marson in [7]). For the sake of completeness, in the Appendix of this paper we give an elementary constructive proof of Proposition 2.1 that follows closely the original idea of Adimurthi, Ghoshal and Veerappa Gowda [1, 2]. Let us stress that both approaches [7] and [1, 2] were developed in a much more technical context of control by initial and boundary data in a bounded domain; in our simpler setting, we were able to drop assumptions such as uniform convexity of $f$ and superlinear growth of $f^{\prime}$ at infinity.

In $[1,2]$ the authors characterize the set of admissible target profiles for a scalar conservation law (6) with uniformly convex flux $f$ as

$$
\begin{aligned}
\mathcal{A}_{T}(J, f)= & \left\{u \in L^{\infty}(J): \exists \rho: J \rightarrow \mathbb{R},\right. \text { right continuous, nondecreasing } \\
& \text { such that } \left.f^{\prime}(u)=\frac{x-\rho(x)}{T}\right\},
\end{aligned}
$$

for some fixed $T>0$ and some bounded interval $J=\left[C_{1}, C_{2}\right]$ in $\mathbb{R}$. In the paper, we write $\mathcal{A}_{T}$ for $\mathcal{A}_{T}(J, f)$ whenever the choice of the interval $J$ and the flux function $f$ is not ambiguous.

A solution $u$ and an initial condition $u_{0}$ that give rise to the target state $u_{T} \in \mathcal{A}_{T}$ at time $t=T$ were obtained in [1] as strong limits in $L_{l o c}^{1}$ of two sequences of piecewise continuous functions $\left(u_{n}\right)_{n}$ and $\left(u_{0, n}\right)_{n}$. Of course, the backward solution is not unique, but the algorithm presented in [1] allows to construct the approximating sequences in a very explicit way and it is possible to infer some qualitative properties of the limits $u$ and $u_{0}$. In Appendix we present in detail a variation (appeared as a remark in [20]) of the original algorithm in [1] with the additional important property that the limit function $u$ is an isentropic solution of $(6)$ in $] 0, T[\times \mathbb{R}$.

Remark 2.1. The regularity of the backward solution $u$ is crucial in the next sections as we aim to solve a continuity equation whose coefficient is $g \circ u$. Beyond the fact that $u$ is isentropic, it is clear that the finer regularity of $u$ is strongly related to the assumptions we make on the flux function $f$.

(i) If the flux function $f$ is merely strictly convex the sequence $u_{n}$ is bounded in $L^{\infty}([0, T] \times \mathbb{R})$, uniformly with respect to $n$. This is a natural consequence of our hypothesis on $f$ and the definition of $\mathcal{A}_{T}$. Basically the function $\left(f^{\prime}\right)^{-1}$ is continuous and in the definition (54) the arguments of $\left(f^{\prime}\right)^{-1}$ are bounded, see the estimate (58). We stress that the function $u$ will not be Lipschitz in general in a strip of the form $(\delta, T-\delta) \times \mathbb{R}, \delta>0$. Consider as an example the case $f(u)=u^{4} / 4$ and take as target state $u_{T}$ the step function $\chi_{(-\infty, 0)}-\chi_{(0, \infty)}$. The backward solution $u$ will consist of a compression wave focusing at $(0, T)$ and we can immediately see from its explicit form

$$
u(x, t)= \begin{cases}1 & \text { for } x \leq-(T-t), \\ \left(\frac{x}{T-t}\right)^{1 / 3} & \text { for }|x| \leq(T-t), \\ -1 & \text { for } x \geq(T-t),\end{cases}
$$


that $u$ is not a Lipschitz function.

(ii) Under the stronger assumption (5) the functions $u_{n}$ are Lipschitz continuous with respect to both variables on $[\delta, T-\delta] \times \mathbb{R}$, for all $\delta>0$, uniformly in $n$, so the limit $u$ is in $W_{\text {loc }}^{1, \infty}([0, T] \times \mathbb{R})$. This regularity is the maximal one can reasonably expect, because in general, the functions $u_{n}$ contain rarefaction and compression waves focusing at $t=0$ and at $t=T$, respectively.

\subsection{On the structure of the set $\mathcal{A}_{T}$}

Let $f$ be a fixed strictly convex flux function, $J=\left[C_{1}, C_{2}\right]$ a bounded interval in $\mathbb{R}$ and $T>0$ a fixed positive time. In this section we discuss some fine properties of the structure of the set $\mathcal{A}_{T}(J, f)$, defined as in (8).

Lemma 2.1. The following statements hold.

(i). $\mathcal{A}_{T}(J, f)$ is a closed set in the strong topology of $L^{1}(J)$.

(ii). $\mathcal{A}_{T+\delta}(J, f) \subset \mathcal{A}_{T}(J, f)$, for all $\delta>0$.

(iii). The set $\mathcal{A}_{T+}(J, f):=\bigcup_{\delta>0} \mathcal{A}_{T+\delta}(J, f)$ is dense in $\mathcal{A}_{T}(J, f)$ in the strong topology of $L^{1}(J)$ (if $J$ is bounded) or $L_{l o c}^{1}(J)$ (if $J$ is unbounded).

Proof. (i). Let $\left(u_{n}\right)_{n}$ be a sequence in $\mathcal{A}_{T}(J, f)$, converging to $\bar{u}$ in the strong topology of $L^{1}(J)$. From the definition of $\mathcal{A}_{T}(J, f)$, (8), we infer the existence of a sequence of nondecreasing, right continuous functions, $\left(\rho_{n}\right)_{n}$, given by

$$
\rho_{n}(x)=x-T f^{\prime}\left(u_{n}(x)\right) .
$$

It is clear that $\rho_{n}$ converges in $L^{1}$ to some nondecreasing function $\rho$, and without loss of generality we can choose $\rho$ to be a right continuous function. The continuity of $f^{\prime}$ and equation (10) allow us to conclude.

(ii). Let $u_{T+\delta} \in \mathcal{A}_{T+\delta}(J, f)$, then there exists some nondecreasing, right continuous function $\rho(x)$ such that $u_{T+\delta}(x)=\left(f^{\prime}\right)^{-1}\left(\frac{x-\rho(x)}{T+\delta}\right)$. Define

$$
\begin{aligned}
\bar{\rho}(x) & =x-T f^{\prime}\left(u_{T+\delta}(x)\right) \\
& =\left(1-\frac{T}{T+\delta}\right) x+\rho(x) \frac{T}{T+\delta},
\end{aligned}
$$

hence $\bar{\rho}$ is a nondecreasing right continuous function in $L^{\infty}(J)$, which implies $\frac{x-\bar{\rho}(x)}{T}=u_{T+\delta} \in A_{T}(J, f)$.

(iii). From (i) and (ii), it becomes clear that the closure of $\mathcal{A}_{T+}(J, f)$ in the $L^{1}$ topology is a subset of $\mathcal{A}_{T}(J, f)$. Consider the semigroup $S^{\rightarrow}: \mathbb{R}^{+} \times L^{\infty}(\mathbb{R}) \rightarrow$ $L^{\infty}(\mathbb{R})$ whose orbits are solutions to Cauchy problems associated to the equation (6). We denote by $S_{\delta}\left(\mathcal{A}_{T}\right)$ the set of profiles which we obtain after time $\delta>0$ by taking the functions in $\mathcal{A}_{T}(J, f)$ as initial data for the Cauchy problem. Let $u_{T} \in \mathcal{A}_{T}(J, f)$, then $S_{\delta}\left(u_{T}\right)$ is in $\mathcal{A}_{T+\delta}(K, f)$, for some interval $K$ such that $J \subset K$. It is clear from definition (8) that $\mathcal{A}_{T}\left(J_{1}, f\right) \subset \mathcal{A}_{T}\left(J_{2}, f\right)$ as soon as $J_{2} \subset J_{1}$. Therefore, for any positive $\delta, S_{\delta}\left(u_{T}\right)$ is in $\mathcal{A}_{T+\delta}(J, f)$. By the $L_{l o c}^{1}$ continuity in time of the semigroup $\delta \mapsto S_{\delta}$, we obtain

$$
\lim _{\delta \rightarrow 0}\left\|S_{\delta}\left(u_{T}\right)-u_{T}\right\|_{L^{1}(\tilde{J})} \rightarrow 0 \text { as } \delta \rightarrow 0
$$


for all bounded subinterval $\tilde{J}$ of $J$. Hence the claim of (iii) follows.

Remark 2.2. For any fixed strictly convex flux function $f$, any bounded interval $J=\left[C_{1}, C_{2}\right]$ in $\mathbb{R}$, any positive time $T$ and any $\delta>0$, there exists $u \in \mathcal{A}_{T}(J, f)$ such that $u \notin \mathcal{A}_{T+\delta}(J, f)$.

Proof. If the flux function $f$ was $C^{2}$ and uniformly convex then, by Oleinik inequality, we would just need to find a profile $u$ such that $\frac{1}{(T+\delta) f^{\prime \prime}(u)}<\partial_{x} u \leq \frac{1}{T f^{\prime \prime}(u)}$. In the strictly convex case we exploit the definition of attainable set, (8). Let $0<\alpha<\frac{\delta}{T+\delta}$, then define $\rho(x)=\alpha x$ and $u=\left(f^{\prime}\right)^{-1}\left(\frac{x-\rho(x)}{T}\right) \in \mathcal{A}_{T}$. If $u$ was in $\mathcal{A}_{T+\delta}$ then we could find some nondecreasing, right continuous function $\bar{\rho}$, such that $f^{\prime}(u)=\frac{x-\bar{\rho}(x)}{T+\delta}=\frac{x-\alpha x}{T}$. Then $\bar{\rho}(x)=x\left[\frac{\delta}{T}(\alpha-1)+\alpha\right]$, which contradicts the fact that $\bar{\rho}$ is nondecreasing. This proves $u \notin \mathcal{A}_{T+\delta}(J, f)$.

\section{Systems endowed with entropies}

In order to keep our presentation as light as possible, we assume that $m=2$ in (1); i.e., we will study the system

$$
\left\{\begin{array}{l}
u_{t}+f(u)_{x}=0 \\
v_{t}+(g(u) v)_{x}=0
\end{array}\right.
$$

where $v$ is a scalar unknown. For isentropic solutions of (14) we construct a theory analogous to the theory of strong entropy solutions of the classical Keyfitz-Kranzer system, see [30], see also [18]; the key points of our analysis are Proposition 2.1(ii), the calculation of entropies for (14) and reduction to the framework of generalized solutions to continuity equations developed in [31]. The study of multi-component systems (1) $(m \geq 2)$ can be done in the same way. In conclusion, we will point out a way to include the Keyfitz-Kranzer system in our analysis.

\subsection{Entropies for a system in the form (14)}

In this section we describe the structure of the entropies for the system (14). Consider $f, g$ restricted to an interval $[a, b]$. We have the following result.

Lemma 3.1. If the system (14) is strictly hyperbolic for $(u, v) \in[a, b] \times \mathbb{R}$, then all its smooth entropies are of the form

$$
\mathcal{E}(u, v)=\eta(u)+e^{-H(u)} \mu\left(v e^{H(u)}\right),
$$

where $H$ is a primitive of the function $u \mapsto \frac{-g^{\prime}(u)}{f^{\prime}(u)-g(u)}$ and $\eta$ and $\mu$ are arbitrary smooth functions. The corresponding entropy flux is given by

$$
\mathcal{Q}(u, v)=q(u)+g(u) e^{-H(u)} \mu\left(v e^{H(u)}\right), \quad q(u)=\int f^{\prime}(u) \eta^{\prime}(u) d u .
$$

Remark 3.1. We notice that the strict hyperbolicity of system (14) ensures that the map $A: u \mapsto e^{-H(u)}$ is well defined on the whole range of values of $u$. The result 
above remains true even if the system is not strictly hyperbolic, provided that the function $H$ is well defined, which means that

$$
u \mapsto \frac{-g^{\prime}(u)}{f^{\prime}(u)-g(u)} \text { is integrable in the range of relevant values of } u \text {. }
$$

The latter condition is satisfied if the singularity at a point $u_{*}$ arising from resonance $\left(f^{\prime}\left(u_{*}\right)=g\left(u_{*}\right)\right)$ in $(17)$ is integrable, but also when the range $\left[\inf _{\mathbb{R}} u_{0}, \sup _{\mathbb{R}} u_{0}\right]$ of values of the initial datum $u_{0}$ does not contain resonance points $u_{*}$.

Proof. We start by computing the Riemann invariants $\left(\omega_{1}, \omega_{2}\right)$ of the system (14) under the assumption that the solution $U=(u, v)$ is smooth. We obtain $\omega_{1}=u$ and $\omega_{2}=v e^{H(u)}=: w$, so that system rewrites (14) as

$$
\left\{\begin{array}{l}
u_{t}+\lambda_{1}(u) u_{x}=0 \\
w_{t}+\lambda_{2}(u) w_{x}=0
\end{array}\right.
$$

with eigenvalues $\lambda_{1}(u)=f^{\prime}(u), \lambda_{2}(u)=g(u)$. Due to property (17), the change of unknowns $(u, v) \leftrightarrow\left(u, e^{H(u)} v\right) \equiv(u, w)$ is bijective, therefore we can look for entropies under the form $\mathcal{E}(u, v)=\widetilde{\mathcal{E}}(u, w)$. Finding entropies means deriving from (18) the scalar conservation law

$$
\widetilde{\mathcal{E}}(u, w)_{t}+\widetilde{\mathcal{Q}}(u, w)_{x}=0
$$

with some entropy-flux $\widetilde{\mathcal{Q}}$. This implies that a smooth entropy / entropy-flux pair has to satisfy the equations

$$
\widetilde{\mathcal{Q}}_{u}(u, w)=\lambda_{1}(u) \widetilde{\mathcal{E}}_{u}(u, w), \quad \widetilde{\mathcal{Q}}_{w}(u, w)=\lambda_{2}(u) \widetilde{\mathcal{E}}_{w}(u, w) .
$$

A standard manipulation (differentiate the first equation with respect to $w$, the second equation with respect to $u$ and equate the mixed second derivatives of the entropy flux) shows that the entropy actually satisfies the equation

$$
g^{\prime}(u) \widetilde{\mathcal{E}}_{w}(u, w)+\left(g(u)-f^{\prime}(u)\right) \widetilde{\mathcal{E}}_{u w}(u, w)=0 .
$$

It is clear that any function with the structure (15) satisfies the equation above. Let us show that they are the only ones. We call $P(u, w)=\widetilde{\mathcal{E}}_{w}(u, w)$ and, thanks to the strict hyperbolicity, we write

$$
P_{u}(u, w)=-H^{\prime}(u) P(u, w),
$$

so that we can integrate the equation above. We get $\widetilde{\mathcal{E}}_{w}(u, w)=e^{-H(u)} \nu(w)$, and then $\widetilde{\mathcal{E}}(u, w)=\eta(u)+e^{-H(u)} \mu(w)$, where $\nu, \eta$ are arbitrary smooth functions and $\mu$ is a primitive of $\nu$. Finally, $\mathcal{E}(u, v)=\eta(u)+e^{-H(u)} \mu\left(v e^{H(u)}\right)$, as we claimed. Formula (16) for the associated entropy-flux $\mathcal{Q}$ follows from relations (19).

Remark 3.2. It is interesting to ask under which conditions the entropy $\mathcal{E}$ is convex, even if this is not a central question in the present work, as all the solutions we consider are isentropic. To answer this question we consider the products $r_{i}^{t} D^{2} \mathcal{E} r_{i}$, $i=1,2$, where $r_{i}$ denotes, as usual, the $i$-th right eigenvector of the system, in particular,

$$
r_{1}=\frac{1}{\sqrt{1+\left(H^{\prime}(u) v\right)^{2}}}\left(\begin{array}{c}
1 \\
-H^{\prime}(u) v
\end{array}\right), \quad r_{2}=\left(\begin{array}{l}
0 \\
1
\end{array}\right) .
$$


It easy to see that $r_{2}^{t} D^{2} \mathcal{E} r_{2}$ is strictly positive if and only if $\mu$ is a convex function of $\omega_{2}$. Next, we have

$$
\begin{aligned}
& r_{1}^{t} D^{2} \mathcal{E} r_{1}= \\
& \frac{1}{1+\left(H^{\prime} v\right)^{2}}\left[\eta^{\prime \prime}+\left(\left(H^{\prime}\right)^{2}-H^{\prime \prime}\right)\left(\mu\left(v e^{H}\right) e^{-H}-\mu^{\prime}\left(v e^{H}\right) v\right)\right] .
\end{aligned}
$$

By using the convexity of $\mu$ and assuming (without loss of generality) that $\mu(0)=0$, it is easy to see that the term $\mu\left(v e^{H(u)}\right) e^{-H(u)}-\mu^{\prime}\left(v e^{H(u)}\right) v$ is negative. Then, in order to get a strictly positive quantity we have to make assumptions on $\eta$ and $H$. We can remark, in particular, that the assumption that $\eta$ is convex will not be sufficient alone. A sufficient condition would be $\eta$ convex and $A: u \mapsto e^{-H(u)}$ concave, but this is far from being necessary.

We end this paragraph with the following elementary observation. Assume $u, v$ are smooth; then function $v$ solves $v_{t}+(g(u) v)_{x}=0$ if and only if

$$
v=A(u) w \text { where } w \text { solves }(A(u) w)_{t}+(g(u) A(u) w)_{x}=0 .
$$

The next paragraph contains technical tools needed to extend the reformulation of (24) to weak solutions $v$, under the assumption that $u$ is an isentropic solution to scalar conservation law $u_{t}+f(u)_{x}=0$ and making the choice $w=e^{H(u)} v$, $A(u)=e^{-H(u)}$ inspired by the result of Lemma 3.1.

\subsection{Strong generalized solutions for continuity equations}

Equation $v_{t}+(g(u) v)_{x}=0$ contained in system (14), and its reformulation (24) are continuity equations. In both cases, coefficients $g(u(t, x))$ (respectively, $A(u(t, x))$ and $B(u(t, x)):=g(u(t, x)) A(u(t, x)))$ do not need to be smooth functions of $(t, x)$. In this paragraph, we explain that the form (24) is preferable because, due to the definition of $A$, the field $(A(u(t, x)), B(u(t, x)))$ on $\mathbb{R}_{+} \times \mathbb{R}$ is divergence-free whenever $u$ is an isentropic solution of $u_{t}+f(u)_{x}=0$.

Recall that transport equation $w_{t}+a(t, x) w_{x}=0$ and its adjoint, the continuity equation $w_{t}+(a(t, x) w)_{x}=0$, can be solved by the method of characteristics provided the coefficient $a$ is Lipschitz continuous; in this case, one has existence and uniqueness of weak solutions for all $L^{\infty}$ initial (or terminal) data, and the solutions are time-reversible. To relax regularity assumptions on $a$ without loosing well-posedness property and time-reversibility property, the notion of renormalized solutions has been put forward in the classical work by DiPerna and Lions [16]. The uniqueness proof for renormalized solutions has undergone major extentions in recent years, see, e.g., [3, 4, 6]. Yet in these theories of renormalized solutions, some regularity assumptions are still needed on $a(t, x)$ and on $\operatorname{div}_{x} a(t, x)$ (typically, a $W^{1, p}$ or a $B V$ regularity of $a$, and a uniform bound of the divergence of $a$ ). We refer to [15] for an extensive account on known results in this direction.

When dealing with equations in one space dimension, as we do in this paper, it is convenient to consider continuity equations of the form (24). Indeed, in the paper [31], Panov considered the problem

$$
\left\{\begin{array}{l}
(A w)_{t}+(B w)_{x}=0 \\
A(0, x) w(0, x)=A(0, x) w_{0}(x)
\end{array}\right.
$$


under the assumptions

- $A$ and $B$ in $L^{\infty}\left(\mathbb{R}_{+} \times \mathbb{R}\right)$;

- $A_{t}+B_{x}=0$ in $\mathcal{D}^{\prime}((0,+\infty) \times \mathbb{R})$;

- there exists $N: \mathbb{R} \rightarrow \mathbb{R}$ such that $\varepsilon N(\varepsilon) \rightarrow 0$ as $\varepsilon$ tends to zero and for all $\varepsilon>0,|B| \leq N(\varepsilon)(A+\varepsilon)$ a.e. in $(0,+\infty) \times \mathbb{R} ;$

- ess $\lim _{t \rightarrow 0^{+}} A(t, x)=A(0, x)$ in $L_{l o c}^{1}$ and $A(0, x)$ belongs to $L^{\infty}(\mathbb{R})$.

For the sake of simplicity, we will require $A \in C\left(\mathbb{R}_{+} ; L_{l o c}^{1}(\mathbb{R})\right)$, which simplifies the statements concerning existence of strong traces of $A w$; in particular, the restrictions $A(t, \cdot)$ are well defined for all $t \geq 0$. Indeed, we will use the theory of [31] with $A=e^{-H(u)}$ where $u \in C\left(\mathbb{R}_{+} ; L_{l o c}^{1}(\mathbb{R})\right.$ is an entropy solution of a scalar conservation law.

Panov showed in [31] that for any given bounded initial condition $w_{0}$ there exists a bounded function $w$, called generalized solution of (25) such that for any test function $\phi$ in $C_{0}^{\infty}([0,+\infty) \times \mathbb{R})$

$$
\int_{0}^{+\infty} \int_{\mathbb{R}}(A w) \phi_{t}+(B w) \phi_{x} d x d t+\int_{\mathbb{R}} A(0, x) w_{0}(x) \phi(0, x) d x=0 .
$$

Moreover, every generalized solution $w$ enjoys the following properties

Strong trace: ess $\lim _{t \rightarrow 0^{+}} A(t, x) w(t, x)=A(0, x) w_{0}(x)$ in $L_{l o c}^{1}(\mathbb{R})$; and for all $T>0$, there exists ess $\lim _{t \rightarrow T^{-}} A(t, x) w(t, x)$ in $L_{l o c}^{1}(\mathbb{R})$.

Reversibility: if $w$ is a generalized solution of (25) and the equality $A(T, x) w(T, x)=$ $A(T, x) w_{T}(x)$ holds in the sense of strong traces, then $t \mapsto w(T-t)$ is a generalized solution to the same equation with $B$ changed into $-B$ and with initial datum $w_{T}$.

Uniqueness: if $A(0, x) w_{0}(x)=0$ a.e. on $\mathbb{R}$ then $A(t, x) w(t, x)=0$ a.e. on $\mathbb{R}_{+} \times \mathbb{R}$.

Renormalization: for any function $\mu$ in $C(\mathbb{R})$ the function $\mu \circ w$ satisfies

$$
\left\{\begin{array}{l}
(A(\mu \circ w))_{t}+(B(\mu \circ w))_{x}=0 \\
(\mu \circ w)(0, x)=\mu\left(w_{0}(x)\right)
\end{array}\right.
$$

in the sense of (26).

This means that in the setting of [31], uniqueness, renormalization and reversibility properties are automatically guaranteed for all generalized solutions. In particular, changing $t$ into $T-t$ and $B$ into $-B$, we have the following corollary.

Corollary 3.1. For every bounded terminal datum $w_{T}$ there exists a unique solution of

$$
\left\{\begin{array}{l}
(A w)_{t}+(B w)_{x}=0 \\
A(T, x) w(T, x)=A(T, x) w_{T}(x)
\end{array}\right.
$$

in the sense

$$
\begin{aligned}
\int_{0}^{+\infty} \int_{\mathbb{R}}(A w) \phi_{t} & +(B w) \phi_{x} d x d t \\
& +\int_{\mathbb{R}} A(0, x) w(0, x) \phi(0, x) d x=\int_{\mathbb{R}} A(T, x) w_{T}(x) \phi(0, x) d x
\end{aligned}
$$


for all $\phi \in C_{0}^{\infty}([0, T] \times \mathbb{R})$, where $A(0, \cdot) w(0, \cdot)$ is the strong initial trace of Aw; this solution possesses the renormalization property and it is reversible, namely, it is the unique weak solution of $(25)$ with initial datum defined by $w_{0}:=w(0, \cdot)$.

\subsection{Main result on non-resonant systems}

According to the analysis of Lemma 3.1, isentropic solutions on $(0, T) \times \mathbb{R}$ for nonresonant systems (14) are weak solutions which satisfy

$$
\mathcal{E}(u, v)_{t}+\mathcal{Q}(u, v)_{x}=0 \text { in } \mathcal{D}^{\prime}((0, T) \times \mathbb{R}),
$$

where $\mathcal{E}, \mathcal{Q}$ take the form (15), (16), respectively. According to the standard admissibility paradigm for hyperbolic systems of conservation laws, isentropic solutions are considered as admissible since they satisfy entropy (in)equalities for all convex entropy $\mathcal{E}$. Then, if for a fixed time $T$, the target state $U_{T}$ is attainable by an isentropic solution, we know that $U_{T}$ is attainable by an admissible solution.

Thus, a result of attainability by isentropic solutions of (14) for a terminal state $U_{T}=\left(u_{T}, v_{T}\right)$ at given time $T$ would imply attainability by admissible solutions. Here, we prove such result.

Theorem 3.1. Assume that $f$ is strictly convex. Assume that the system (14) is non-resonant for the range $[a, b]$ of values of $u$, i.e., for all $u \in[a, b], f^{\prime}(u) \neq$ $g(u)$. Given $T>0$, take a $[a, b]$-valued terminal datum $u_{T}$ that belongs to the set $\mathcal{A}_{T}(\mathbb{R}, f)$ of states attainable at time $T$ by entropy solutions of $u_{t}+f(u)_{x}=0$; take $v_{T} \in L^{\infty}(\mathbb{R})$. Then there exists a unique initial datum $U_{0}=\left(u_{0}, v_{0}\right)$ such that (14) admits a unique isentropic solution $U=(u, v)$ on $(0, T) \times \mathbb{R}$ with $U(0, \cdot)=U_{0}$ and $U(T, \cdot)=U_{T}$.

On the contrary, whenever $u_{T} \notin \mathcal{A}_{T}(\mathbb{R}, f)$, for any $v_{T} \in L^{\infty}(\mathbb{R})$ the terminal state $U_{T}=\left(u_{T}, v_{T}\right)$ is not attainable at time $T$ by any entropy solution of (14).

Proof. Recall that since $f$ is strictly convex, according to Proposition 2.1, every $u_{T} \in \mathcal{A}_{T}$ gives rise to a unique initial datum $u_{0}$ such that $u_{0}=u(0, \cdot)$ and $u_{T}=$ $u(T, \cdot)$ are connected by the trajectory $u: t \in[0, T] \mapsto L^{\infty}(\mathbb{R})$ which represents an isentropic, reversible solution to $u_{t}+f(u)_{x}=0$. In addition, $u$ takes values in $[a, b]$ due to the maximum principle for entropy solutions. Being isentropic solution means that for any entropy, entropy-flux pair $(\eta, q)$ (with non necessarily convex $\eta$ ) we have

$$
\eta(u)_{t}+q(u)_{x}=0 \text { in } \mathcal{D}^{\prime}((0, T) \times \mathbb{R}) .
$$

The functions $\eta: u \mapsto A(u)=e^{-H(u)}$ and $q: u \mapsto B(u)=g(u) A(u)$ do constitute such an entropy / entropy-flux pair, thanks to the definition of $H$ in Lemma 3.1; therefore $A(u)_{t}+B(u)_{x}=0$ in the sense of distributions. Moreover, $A(u) \geq$ const $>0$ for all $u \in[a, b]$, and $|B(u)| \leq\|g\|_{C([a, b])} A(u)$. Therefore we are in a simplified version (without degeneracy of $A$ ) of the generalized continuity equation setting considered by Panov in [31] and recalled in $\S 3.2$. Applying the result of Corollary 3.1 , we see that for any given terminal condition $v_{T}$ in $L^{\infty}(\mathbb{R})$, there exists a unique function $v$ bounded weak solution of

$$
\left\{\begin{array}{l}
v_{t}+(g(u) v)_{x}=0 \\
v(T, x)=v_{T}(x)
\end{array}\right.
$$


such that $v(t, x)$ is the product $A(t, x) w(t, x)$, where $w$ solves (28) in the sense (29) with terminal condition $w_{T}(x)=e^{-H\left(u_{T}(x)\right)} v_{T}(x)$.

Consider now the bounded vector valued function $U=(u, v)$. From the definitions of $u$ and $v$, and the renormalization property for $w$ we immediately obtain that $U$ is an isentropic solution of the system (14) with terminal datum $\left(u_{T}, v_{T}\right)$. Indeed, for all Lipschitz functions $\eta$ and $\mu$ on $[a, b]$ we have the entropy inequality (31) for $u$ along with the renormalization property

$$
(A \mu(w))_{t}+(B(u) \mu(w))_{x}=0 \text { in } \mathcal{D}^{\prime}((0, T) \times \mathbb{R})
$$

for $w$. Combining the two and taking into account the result of Lemma 3.1 and the definition of $A$, we readily reach to the property (30) for general entropies $\mathcal{E}$ of (14).

Finally, let us justify the non-attainability result for $u_{T} \notin \mathcal{A}_{T}$. If $U=(u, v)$ is an entropy solution of system (14), then using the entropy $\mathcal{E}(u, v):=\eta(u)$ with convex $\eta$ we find that $u$ should be an entropy solution of the scalar conservation law. Then, the fact that data $U_{T}=\left(u_{T}, v_{T}\right)$ with $u_{T} \notin \mathcal{A}_{T}$ are not reacheable by entropy solutions of (14) follows readily from Proposition 2.1.

Remark 3.3. In this note we were interested in the attainability problem; but, naturally, we have treated in passing the existence problem. Indeed, from the arguments put forward in this section it follows that, given $T>0$, for any datum $\left(u_{0}, v_{0}\right) \in \mathcal{A}_{T}(\mathbb{R},-f) \times L^{\infty}(\mathbb{R})$ there exists a unique isentropic solution on $[0, T] \times \mathbb{R}$ of the Cauchy problem $(14),\left.(u, v)\right|_{t=0}=\left(u_{0}, v_{0}\right)$.

Example 1. The system of two component chromatography describes the evolution of the (nonegative) concentrations of two solutes, $u_{1}$ and $u_{2}$,

$$
\left\{\begin{array}{l}
\partial_{t} u_{1}+\partial_{x}\left(\frac{u_{1}}{1+u_{1}+u_{2}}\right)=0, \\
\partial_{t} u_{2}+\partial_{x}\left(\frac{u_{2}}{1+u_{1}+u_{2}}\right)=0 .
\end{array}\right.
$$

Passing to the variables $v=u_{1}+u_{2}, w=u_{1}-u_{2}$, the system becomes

$$
\left\{\begin{array}{l}
\partial_{t} v+\partial_{x}\left(\frac{v}{1+v}\right)=0, \\
\partial_{t} w+\partial_{x}\left(\frac{w}{1+v}\right)=0,
\end{array}\right.
$$

which has the form (14) with $f(v)=\frac{v}{1+v}$ and $g(v)=\frac{1}{1+v}$, defined on $\mathcal{I}:=$ $(-1,+\infty)$. It is easy to check that $f$ is convex and $f(v) \neq g^{\prime}(v)$ on $\mathcal{I}$, therefore the result of Theorem 3.1 applies to the system in this form. A detailed study of the well-posedness for the two components chromatography system, which exploits the change of variables above, can be found in [5].

\subsection{On the Keyfitz-Kranzer system}

Consider the one-dimensional Keyfitz-Kranzer system, i.e. a system of the form

$$
U_{t}+(\phi(|U|) U)_{x}=0,
$$

where $\phi$ is a smooth function from $\mathbb{R}_{+}$to $\mathbb{R}$ such that $\lim _{r \rightarrow 0+} r \phi(r)=0$ and $U(t, x) \in \mathbb{R}^{m+1}, m \geq 1$. System (36) has first been introduced as the prototype of 
non-strictly hyperbolic system, see [26, 14]. This system does not have form (1), but it can be treated with minor adaptations. Moreover, the general case we treated above took inspiration from the Keyfitz-Kranzer case: see Remark 3.4.

Indeed, system (36) is formally equivalent to the system

$$
\left\{\begin{array}{l}
r_{t}+(\phi(r) r)_{x}=0 \\
(r w)_{t}+(\phi(r) r w)_{x}=0
\end{array}\right.
$$

under the additional constraint $|w|=1$ (being understood that $\left|w_{0}\right|=1$ ), where the variables $(r, w)$ have the sense $r=|U|$ and $w=U /|U|$. In the Keyfitz-Kranzer setting, $r \mapsto \phi(r) r$ need not be assumed strictly convex; nonetheless, the latter system would be quite similar to systems of our family (1) if the constraint $|w|=1$ followed automatically from $\left|w_{0}\right|=1$. Such constraint-preservation property is a particular kind of renormalization property for continuity equations; for the above system (37), it actually holds true provided the appropriate notion of solution is considered. Such appropriate notion of strong generalized entropy solution for the Cauchy problem associated to system (36) has been introduced in the papers by Freistühler, [18], and Panov, [30]. The Cauchy problem for (36) is well posed in the setting of strong generalized entropy solutions. Here we give a version of the definition that is intimately related to the setting of $\S 3.2$.

Definition 3.1. Let $U_{0} \in L^{\infty}\left(\mathbb{R} ; \mathbb{R}^{m}\right)$ be initial condition imposed to the system (36). A function $U \in L^{\infty}\left((0, T) \times \mathbb{R} ; \mathbb{R}^{m}\right)$ is a strong generalized entropy solution for the system (36) if

- the function $r(t, x)=|U(t, x)|$ is the Kruzhkov entropy solution of

$$
\begin{cases}r_{t}+(r \phi(r))_{x}=0, & (t, x) \in(0, T) \times \mathbb{R} \\ r(0, x)=\left|U_{0}(x)\right|, & x \in \mathbb{R}\end{cases}
$$

- the function $U$ satisfies $U=r w$, where $w$ is the solution of (25) in the weak sense (26) with initial datum $w_{0}=U_{0} /\left|U_{0}\right|$ and the coefficients $A=r(t, x)$, $B=r(t, x) \phi(r(t, x))$.

In the above definition, the value of $U_{0}(x) /\left|U_{0}(x)\right|$ can be taken arbitrary (e.g., in the unit $m$-dimensional sphere $\left.\mathbb{S}^{m}\right)$ at the points where $U_{0}(x)=0$.

As an outcome of the theory of [31] for weak solutions of (25), the definition above is equivalent to the definition of renormalized entropy solution introduced in $[6,4]$ and used in $[19,5]$ :

Definition 3.2. The function $U \in L^{\infty}\left((0, T) \times \mathbb{R} ; \mathbb{R}^{m}\right)$ is a renormalized entropy solution for the system (36) if

- the function $r(t, x)=|U(t, x)|$ is the entropy solution of

$$
\begin{cases}r_{t}+(r \phi(r))_{x}=0, & (t, x) \in(0, T) \times \mathbb{R} \\ r(0, x)=\left|U_{0}(x)\right|, & x \in \mathbb{R} ;\end{cases}
$$

- for any test function $\psi \in C_{0}^{\infty}([0, T) \times \mathbb{R})$, and any continuous function $\mu$ : $\mathbb{S}^{m} \mapsto \mathbb{R}, \mathbb{S}^{m}$-valued function $w=U /|U|$ verifies

$$
\int_{0}^{T} \int_{\mathbb{R}} r \mu(w) \psi_{t}+\phi(r) r \mu(w) \psi_{x} d x d t+\int_{\mathbb{R}} r(0, x) H(w(0, x)) \psi(0, x) d x=0 .
$$


The use of Definition 3.2 is simplified if the data are taken away from the origin which is the umbilical point for the system, i.e., $\left|U_{0}\right| \geq \delta>0$ (see [19]). In both cases, the renormalization property for $w=U /|U|$ (explicitly stated in Definition 3.2, implicitly contained in Definition 3.1) and the uniqueness of renormalized solutions for equations of form $(A w)_{t}+(B w)_{x}=0$ lead to the crucial constraint-preservation property: $|w|=1$ a.e. on $\{(t, x) \mid r(t, x)>0\}$ due to $\left|w_{0}\right|=1$ (steaming from the definition $\left.w_{0}=U_{0} /\left|U_{0}\right|\right)$. This property ensures that weak (resp., renormalized) solutions constructed for the component $w$ of the transformed system (37) give rise to strong generalized entropy solutions (resp., renormalized entropy solutions) of the original system (36).

According to the well-posedness results of [31], the question of attainability for the Keyfitz-Kranzer system (36) should be asked in the setting of strong generalized entropy solutions. For the case of Keyfitz-Kranzer system, the following analogue of Theorem 3.1 holds.

Theorem 3.2. Assume that $f: r \mapsto r \phi(r)$ in system (36) is strictly convex on $[a, b] \subset[0,+\infty)$. Given $T>0$, take a terminal datum $U_{T}$ such that $\left|U_{T}\right|$ is $[a, b]$ valued and belongs to the set $\mathcal{A}_{T}(\mathbb{R}, f)$ of states attainable at time $T$ by entropy solutions of $u_{t}+f(u)_{x}=0$. Then there exists a unique initial datum $U_{0}$ such that (14) admits a unique strong generalized entropy solution (or, equivalently, renormalized entropy solution) $U$ on $(0, T) \times \mathbb{R}$ and $U(T, \cdot)=U_{T}$.

On the contrary, whenever $\left|U_{T}\right| \notin \mathcal{A}_{T}(\mathbb{R}, f)$, the terminal state $U_{T}$ is not attainable at time $T$ by any strong generalized entropy solution (or, equivalently, renormalized entropy solution) of Keyfitz-Kranzer system (36).

Proof. The proof follows the lines of the one of Theorem 3.1, the only difference being the fact that the isentropic character of $r$, backward solution of $r_{t}+f(r)_{x}=0$ on $[0, T]$ becomes not important, while the renormalization property for $w$ is now needed not in order to justify that $U$ is an entropy (isentropic) solution of system (14), but in order to ensure that if satisfies the constraint $|w|=1$ and gives rise to the appropriately defined solution of the Keyfitz-Kranzer system (36).

Remark 3.4. The notion of isentropic solution of non-resonant system (14) of the previous paragraph appears as the analogue of the notion of strong entropy solution for the Keyfitz-Kranzer system (36). The main difference is that one has the wellposedness result for strong entropy solutions of (36) with any bounded initial data, while only data $u_{0}$ giving rise to an isentropic solution on $[0, T]$ are suitable in the existence theory outlined in Remark 3.3.

The reason for this difference lies in the fact that the divergence-free relation $A(r)_{t}+B(r)_{x}=0$, essential for application of the theory of [31], holds in the case of (36) for any weak solution of the conservation law $r_{t}+(r \phi(r))_{x}=0$, being $A(r)=r$ and $B(r)=r \phi(r)$; while in the case of systems (14) the analogous relation $A(u)_{t}+$ $B(u)_{x}=0$ is readily guaranteed only for isentropic solutions of the conservation law $u_{t}+f(u)_{x}=0$, A being a nonlinear function of $u$.

To make the similarities between (14) and (36) more apparent, let us recall a lemma due to Frid, [19], on the structure of the entropies for the Keyfiz-Kranzer system, see also [30, Th. 1]. 
Lemma 3.2. All smooth entropies of the system (37) defined for $\delta \leq r \leq M$, for some positive constants $\delta$ and $M$, are of the form

$$
\mathcal{E}(u)=\eta(r)+r \mu(w),
$$

with $r=|u|$ and $w=u /|u|$, for appropriate smooth functions $\eta$ and $\mu$.

The proof given in [19] for Lemma 3.2 inspired our proof of Lemma 3.1.

\section{Resonant systems}

In this section, we study the set of attainable states for system (1) without enforcing the non-resonance assumption.

$$
g(u) \neq f^{\prime}(u) \text { for all } u \in[a, b],
$$

where $[a, b]$ is an interval containing the values of $u_{T}$. When (41) fails, (17) fails and nontrivial (nonlinearly $v$-dependent) entropies of form (3.1) cannot be defined. Our result in this setting is weaker than the one we obtained in the non resonant case, as we show that the set of states which are exactly attainable in a fixed time $T$ is a dense subset of $\mathfrak{A}_{T}=\mathcal{A}_{T} \times L^{\infty}(\mathbb{R})$. Two remarks are here in order

- we are not presenting a formal proof of the fact that $\mathfrak{A}_{T}$ contains states which are not exactly attainable in the resonant case. In this sense our result is not optimal.

- Nevertheless, in view of the applications and, in particular the numerical simulations, we can say that, in the resonant case, $\mathfrak{A}_{T}$ contains states which are difficult to treat in practice. This point is highlited in Section 4.3.

As in Section 3, for the sake of simplicity we will restrict our attention to the case $m=1$.

\subsection{Attainability and approximate attainability result}

When system (14) is resonant we cannot rely on the theory outlined in Section 3.2 to solve the continuity equation for $v$, and we aim to use the well established DiPernaLions theory instead, [16]. Therefore, we have to avoid the situation in which the $u$ component of the solution of system (14) contains rarefaction (respectively, compression) waves focusing at $t=0$ (resp., at $t=T$ ). For this reason, in the sequel we replace $\mathcal{A}_{T}$ of attainable terminal states for $u$ by the smaller but $L_{l o c}^{1}$-dense subset $\mathbb{A}_{T}$ defined below. The isentropic backward solutions for $u_{T} \in \mathbb{A}_{T}$ do not contain rarefaction of compression waves focusing at times $t=0$ or $t=T$ respectively; moreover, under assumption (5) it is easily seen that the corresponding solution $u$ belongs to $W^{1, \infty}([0, T] \times \mathbb{R})$, see Remark 2.1. In this setting, we have the following result.

Theorem 4.1. Consider system (14) under the local uniform convexity assumption (5) on $f$. Let $\mathbb{A}_{T}=W^{1, \infty}(\mathbb{R}) \cap\left(\cup_{\delta>0} \mathcal{A}_{T+\delta}(f, \mathbb{R})\right)$. Then

- every state $U_{T} \in \mathbb{A}_{T} \times L^{\infty}(\mathbb{R})$ is controllable at time $t=T$;

- every state in $U_{T} \in \mathfrak{A}_{T}=\mathcal{A}_{T}(f, \mathbb{R}) \times L^{\infty}(\mathbb{R})$ is approximatively controllable at time $t=T$, with respect to $L_{\text {loc }}^{1}(\mathbb{R})$ topology. 
The corresponding backward solutions $U=(u, v)$ for $U_{T}=\left(u_{T}, v_{T}\right)$ are admissible in the sense that the component $u$ can be chosen to be the unique isentropic solution of the first equation of (14) with terminal datum $u_{T}$ and the component $v$ is the unique DiPerna-Lions renormalized solution of the second equation of (14) with terminal datum $v_{T}$.

Let us stress that the notion of solution admissibility put forward in the above statement is the strongest one that we are able to formulate, having in mind

- the absence of non-trivial entropies of the system (so that the above solution is, in fact, an entropy solution, all entropies being described by $\mathcal{E}(u, v)=\eta(u)+C v)$;

- the choice of the strongest available component-per-component notions of admissibility for weak solutions $U=(u, v)$ of $(14)$.

Proof. For the proof of the first claim, keeping in mind the result of Proposition 2.1 we only need to construct a reversible solution to the continuity equation

$$
v_{t}+(c(t, x) v)_{x}=0 \text { on }[0, T] \times \mathbb{R}
$$

with every given terminal datum $v_{T}$ and $c(t, x)=g(u(t, x))$ with $u$ the isentropic solution of $u_{t}+f(u)_{x}=0$ given by Proposition 2.1 ; by the assumption $u_{T} \in \mathbb{A}_{T}$ and $(5)$, we have $W^{1, \infty}([0, T] \times \mathbb{R})$ regularity of the coefficient $c$. In this framework, uniqueness of a weak solution (which is therefore reversible) for any given $L^{\infty}$ initial (respectively, terminal) datum is ensured. The solution can be constructed by the characteristics method, but it can also be constructed as the unique renormalized solution in the sense of DiPerna and Lions [16] of the initial-value (respectively, of the terminal-value) problem for equation (42).

Further, the second claim follows from the first one, using Lemma 2.1. Indeed, by Lemma $2.1, \mathcal{A}_{T+}=\cup_{\delta>0} \mathcal{A}_{T+\delta}(f, \mathbb{R})$ is dense in $\mathcal{A}_{T}$. Further, if $u_{T} \in \mathcal{A}_{T+\delta}$ for some $\delta>0$, it can be approached in $L_{\text {loc }}^{1}(\mathbb{R})$ by states of the form $S_{h}^{\leftarrow} u_{T}$, where $S^{\leftarrow}$ is the semigroup which trajectories are entropy solutions of (3) (which means, backward solutions of (6)). For $h<\delta / 2, S_{h}^{\leftarrow} u_{T} \in \mathcal{A}_{T+\delta / 2}$ because the trajectory $t \mapsto S_{t}^{\leftarrow} u_{T}$ is an isentropic, and therefore reversible, entropy solution of (6). Since we also have $S_{h}^{\leftarrow} u_{T} \in W^{1, \infty}(\mathbb{R})$ (see Remark 2.1(ii)), the density of $\mathbb{A}_{T}$ in $\mathcal{A}_{T}$ follows. Hence also $\mathbb{A}_{T} \times L^{\infty}(\mathbb{R})$ is dense in $\mathfrak{A}_{T}$, in $L_{\text {loc }}^{1}$ sense.

\subsection{On the relation between results of Theorems 3.1 and 4.1}

It is clear that, whenever $u_{T} \in \mathbb{A}_{T}$, both results of Theorems 3.1 and 4.1 apply. This is interesting because Theorem 3.1 relies on an existence result for generalized continuity equation (25), which proof relies on a rather non-explicit construction or approximation method. For renormalized solutions in the sense of DiPerna and Lions [16] used in the proof of Theorem 4.1, numerical methods were developed. In particular, in the regular-coefficient framework exploited in Theorem 4.1, the algorithm of [23] for more general duality solutions of (42) can be used, see Section 5 .

In this relation, let us observe that solutions constructed in Theorems 3.1 and 4.1 do coincide. Indeed, the following observation holds.

Remark 4.1. Given a field of coefficients $(A, B)$ satisfying the assumptions of $\S 3.2$ and such that $A \geq \delta>0, B=c A$ with Lipschitz regular coefficient $c$, the unique generalized weak solution $w$ of $(A w)_{t}+(B w)_{x}=0$ is such that $v=A w$ is the 
renormalized solution in the sense of DiPerna-Lions of $v_{t}+(c v)_{x}=0$ with initial datum $v_{0}(\cdot)=A(0, \cdot) w_{0}(\cdot)$.

It should be stressed that the relation between the renormalization properties for $w$ and for $v$ is not straightforward, because a nonlinear function of $v=A w$ cannot be reduced to the form $A \mu(w)$. The arguments for the proof of the above lemma were developed in [31, Sect. 7.1].

\subsection{Examples of forward and backward non-uniqueness for the resonant case}

In this Section we propose some elementary examples of states that cannot be considered as attainable in practice.

Example 2. First, consider the fully resonant case $g(u)=f^{\prime}(u)$, for instance, this occurs for system

$$
\left\{\begin{array}{l}
u_{t}+\left(\frac{u^{2}}{2}\right)_{x}=0 \\
v_{t}+(u v)_{x}=0
\end{array}\right.
$$

Here, solutions $v$ corresponding to self-similar $u$ can be easily computed. To be specific, given any $C^{1}$, compactly supported in $(-1,1)$ function $K$ by an explicit calculation we see that $U=(u, v)$ defined by

$$
u(t, x)=\left\{\begin{array}{rl}
1, & x \geq t \\
\frac{x}{t}, & -t \leq x \leq t \\
-1, & x \leq-t
\end{array} \quad v(t, x)=\frac{1}{t} K\left(\frac{x}{t}\right) \equiv\left\{\begin{aligned}
0, & x \geq t \\
\frac{1}{t} K\left(\frac{x}{t}\right), & -t \leq x \leq t \\
0, & x \leq-t
\end{aligned}\right.\right.
$$

gives a family of weak solutions to system (43) that correspond to the same initial data $u_{0}(x)=\operatorname{sign}(x)$ and $v_{0}(x)=0$ and to terminal data $u_{T}=\min \left\{1, \max \left\{-1, \frac{x}{T}\right\}\right\}$ and $v_{T}^{K(\cdot)}=\frac{1}{T} K\left(\frac{x}{T}\right)$. Being regular in $(\delta, T] \times \mathbb{R}$ for all $\delta>0$, assuming strong initial and terminal traces, these solutions should be regarded as admissible solutions to (43) (in particular, these solutions are renormalized solutions in the sense of DiPerna and Lions [16]). Moreover, the characteristics method (or the theory of [16] applied on $[\delta, T]$ for every $\delta>0$ ) ensures that $U$ is the unique backward solution of (43) with terminal data $U_{T}=\left(u_{T}, v_{T}^{K(\cdot)}\right)$. Thus, backward resolution of (43) with different terminal data $\left(\min \left\{1, \max \left\{-1, \frac{x}{T}\right\}\right\}, v_{T}^{K(\cdot)}\right)$ for $K \in C_{0}^{1}((-1,1))$ gives rise to the same initial datum $U_{0}=(\operatorname{sign}(\cdot), 0)$. Then all states $\left(\min \left\{1, \max \left\{-1, \frac{\dot{T}}{T}\right\}\right\}, v_{T}^{K(\cdot)}\right)$ with different functions $K \in C_{0}^{1}((-1,1))$ belong to the image of the system (43) due to explicit solutions given above. However, thanks to the forward non-uniqueness for (43), this information is useless in practice. In particular, it is clear that starting from $U_{0}$, any given approximation procedure will select one and only one controllable terminal datum $v_{T}^{K(\cdot)}$, while other data of the same family will not be attainable in practice.

Observe that, unless $K \equiv 0$, the above admissible solutions belong to the space $L^{\infty}\left((0, T), L^{1}(\mathbb{R})\right)$ but the $L^{\infty}$ norm of $u(t, \cdot)$ blows up, as $t$ approaches zero. Therefore, if one limits the functional framework for $u, v$ to the space $L^{\infty}$, as we have done in the other sections of this paper, the target data (44) would be automatically excluded, except for $K \equiv 0$. 
One might think that the non-uniqueness in the above example is due to the fact that (43) is a fully resonant system. But the same features persist for the following system that is resonant (and actually, not hyperbolic) at $u_{*}=1$ (note that the resonance at $u_{*}=0$ is not an obstacle because (17) holds in a neighbourhood of $\left.u_{*}=0\right)$.

Example 3. Consider the system

$$
\left\{\begin{array}{l}
u_{t}+\left(\frac{u^{2}}{2}\right)_{x}=0 \\
v_{t}+\left(u^{2} v\right)_{x}=0
\end{array}\right.
$$

In this case, given any $C^{1}$, compactly supported in $(0,1)$ function $K$, by an explicit calculation we see that $U=(u, v)$ defined by

$$
u(t, x)=\min \left\{1, \max \left\{0, \frac{x}{t}\right\}\right\} \text { with } v(t, x)=\frac{1}{x^{2}} K\left(\frac{1}{x}-\frac{1}{t}\right)
$$

gives a family of admissible solutions to (45). All the corresponding terminal states $U_{T}=(u(T, \cdot), v(T, \cdot))$ for different $K \in C_{0}^{1}((0,1))$ can be seen as attainable (with solutions $\left.\left.(u, v) \in L^{\infty}((0, T) \times \mathbb{R})\right) \times L^{\infty}\left((0, T), L^{1}(\mathbb{R})\right)\right)$ starting from the same initial datum $U_{0}=(u(0, \cdot), 0)$, due to forward non-uniqueness for (45).

Remark 4.2. One can also observe that setting $\hat{U}=(\hat{u}, \hat{v})$ with

$$
\hat{u}:(t, x) \mapsto u(T-t,-x), \quad \hat{v}:(t, x) \mapsto v(T-t,-x)
$$

where $(u, v)$ are solutions for (43) (respectively, for (45)) from the above families, give a family of admissible weak solutions to (43) (resp., to (45)) on $[0, T] \times \mathbb{R}$ with the same terminal data and with different initial data. This means that backward resolution of the continuity equation on $v$ contained in (14) can be intrinsically non-unique, even if different solutions lead to different controls of the same terminal state. We refer to numerical examples of Section 5 for an illustration of the fact that this phenomenon also complicates the practical construction of controls for resonant systems (14).

\section{$5 \quad$ Numerics}

In this section we present some results on the numerical backward resolution of system (14) on $[\sigma, T] \times J$, where $J=\left[C_{1}, C_{2}\right], 0<\sigma<T$ and where the final data $\left(u_{T}, v_{T}\right)$ belong to $\mathcal{A}_{T} \times L^{\infty}(J)$. We first begin with the description of the numerical method.

\subsection{The numerical method}

Let us recall that the solution $u$ obtained by the algorithm introduced in Section 7.1 , is an isentropic solution of the scalar conservation law. Consequently, we have that $u(t,)=.w(T-t,$.$) where w$ is the unique entropy solution of the auxiliary forward problem

$$
\left\{\begin{array}{l}
w_{t}+(-f(w))_{x}=0 \\
\left.w\right|_{t=0}=u_{T}
\end{array}\right.
$$


Thus the numerical backward resolution of system (14) is based on the numerical resolution of the following auxiliary forward problem:

$$
\left\{\begin{array}{l}
u_{t}+(\widetilde{f}(u))_{x}=0 \\
v_{t}-(g(u) v)_{x}=0
\end{array}\right.
$$

where $\tilde{f}(u)=-f(u)$ and $(u(0,),. v(0,))=.\left(u_{0}, v_{0}\right) \in \mathcal{A}_{T} \times L^{\infty}(J)$. In order to solve (46), we use the finite volume method detailed below.

To perform the computations, we look for a fixed domain by determining the domains of dependence of the conservation law and of the continuity equation. We define

$$
\begin{gathered}
B_{1}^{(1)}=C_{1}+(T-\sigma) \tilde{f}^{\prime}\left(u_{0}\left(C_{1}\right)\right), \quad B_{2}^{(1)}=C_{2}+(T-\sigma) \tilde{f}^{\prime}\left(u_{0}\left(C_{2}\right)\right), \\
B_{1}^{(2)}=C_{1}-(T-\sigma) g\left(u_{0}\left(C_{1}\right)\right), \quad B_{2}^{(2)}=C_{2}-(T-\sigma) g\left(u_{0}\left(C_{2}\right)\right) .
\end{gathered}
$$

We deduce the fixed domain of interest $K=\left[B_{1}, B_{2}\right]$ where $B_{1}=\min \left\{C_{1}, B_{1}^{(1)}, B_{1}^{(2)}\right\}$ and $B_{2}=\max \left\{C_{2}, B_{2}^{(1)}, B_{2}^{(2)}\right\}$. Assuming that in practice $v_{T} \in B V(J)$, we extend the final states as follow

$$
u_{T}(x)=\left\{\begin{array}{ll}
u_{T}\left(C_{1}\right) & \text { if } x \in\left[B_{1}, C_{1}\right] \\
u_{T}\left(C_{2}\right) & \text { if } x \in\left[C_{2}, B_{2}\right]
\end{array} \quad, v_{T}(x)=\left\{\begin{array}{ll}
v_{T}\left(C_{1}\right) & \text { if } x \in\left[B_{1}, C_{1}\right] \\
v_{T}\left(C_{2}\right) & \text { if } x \in\left[C_{2}, B_{2}\right]
\end{array} .\right.\right.
$$

We now introduce a regular grid:

$$
B_{1}=x_{1 / 2}<x_{3 / 2}<\cdots<x_{M-1 / 2}<x_{M+1 / 2}=B_{2} .
$$

We define the cells $K_{j}=\left[x_{j-1 / 2}, x_{j+1 / 2}\right]$, the center of the cells $x_{j}=\frac{1}{2}\left(x_{j-1 / 2}+\right.$ $\left.x_{j+1 / 2}\right)$ and the space step $\Delta x=x_{j+1 / 2}-x_{j-1 / 2}$, where $1 \leq j \leq M$. We define a time discretization

$$
t^{0}=\sigma<t^{1}<\cdots<t^{n}<t^{N+1}=T,
$$

with a constant time step $\Delta t=t^{n+1}-t^{n}$ for $0 \leq n \leq N$. We denote by $u_{j}^{n}$ and $v_{j}^{n}$ the unknowns which are the approximations of the averages of $u\left(t^{n},.\right)$ and $v\left(t^{n},.\right)$ on $K_{j}$ respectively . Namely

$$
u_{j}^{n} \simeq \frac{1}{\Delta x} \int_{K_{j}} u\left(t^{n}, x\right) d x \text { and } v_{j}^{n} \simeq \frac{1}{\Delta x} \int_{K_{j}} v\left(t^{n}, x\right) d x .
$$

We set

$$
u_{j}^{0}=\frac{1}{\Delta x} \int_{K_{j}} u_{0}(x) d x \text { and } v_{j}^{0}=\frac{1}{\Delta x} \int_{K_{j}} v_{0}(x) d x .
$$

Given $\left(u_{j}^{n}, v_{j}^{n}\right)$, we shall compute $\left(u_{j}^{n+1}, v_{j}^{n+1}\right)$. We first update $u_{j}^{n}$ by using a Godounov scheme

$$
u_{j}^{n+1}=u_{j}^{n}-\frac{\Delta t}{\Delta x}\left(\mathcal{F}\left(u_{j}^{n}, u_{j+1}^{n}\right)-\mathcal{F}\left(u_{j-1}^{n}, u_{j}^{n}\right)\right),
$$

where $\mathcal{F}$ is the standard Godounov numerical flux (see e.g. [22, 29]):

$$
\mathcal{F}\left(u_{\ell}, u_{r}\right)= \begin{cases}\min _{u \in\left[u_{\ell}, u_{r}\right]} \tilde{f}(u), & \text { if } u_{\ell} \leq u_{r} \\ \max _{u \in\left[u_{r}, u_{\ell}\right]} \tilde{f}(u), & \text { otherwise. }\end{cases}
$$


Next, we update $v_{j}^{n}$ by using an upwind-type scheme (see [17, 23]). We define

$$
g_{j+1 / 2}^{n}=(1-\theta) g\left(u_{j}^{n}\right)+\theta g\left(u_{j+1}^{n}\right), \quad 0 \leq \theta \leq 1 .
$$

For $s \in \mathbb{R}$, we set $s^{+}=\max (0, s)$ and $s^{-}=\min (s, 0)$. Then we update $v_{j}^{n+1}$ by

$v_{j}^{n+1}=v_{j}^{n}+\frac{\Delta t}{\Delta x}\left\{\left[\left(g_{j+1 / 2}^{n}\right)^{+} v_{j}^{n}-\left(g_{j-1 / 2}^{n}\right)^{+} v_{j-1}^{n}\right]+\left[\left(g_{j+1 / 2}^{n}\right)^{-} v_{j+1}^{n}-\left(g_{j-1 / 2}^{n}\right)^{-} v_{j}^{n}\right]\right\}$.

For the boundary conditions, we set

$$
\left(u_{0}^{n}, v_{0}^{n}\right)=\left(u_{T}\left(C_{1}\right), v_{T}\left(C_{1}\right)\right), \quad\left(u_{M+1}^{n}, v_{M+1}^{n}\right)=\left(u_{T}\left(C_{2}\right), v_{T}\left(C_{2}\right)\right), 1 \leq n \leq N+1 .
$$

\section{Remark 5.1.}

(i) As we already pointed out, $u$ is the entropy solution of the conservation law of (46). Therefore, any standard monotone consistent finite volume method can be used to approximate $u$.

(ii) Let us mention that Gosse and James [23] proved convergence results for the upwind scheme described above, in the more general framework of duality solution for one-dimensional transport and continuity equations (see [8, 9]). They also proved convergence results for Lax-Friedrichs schemes. Therefore these latter schemes are also relevant schemes for the transport and continuity equations.

(iii) Another approach to solve system (46) is to use different grids for the unknowns $u$ and $v$. More precisely, one can look for the pair $\left(u_{j+1 / 2}^{n}, v_{j}^{n}\right)$, where

$$
u_{j+1 / 2}^{n} \simeq \frac{1}{\Delta x} \int_{x_{j}}^{x_{j+1}} u\left(t^{n}, x\right) d x .
$$

Then, one can define $g_{j+1 / 2}^{n}=g\left(u_{j+1 / 2}^{n}\right)$. However, the numerical experiments we have performed with this second method, seem to give less good results.

(iv) For the numerical simulations, we always take $\theta=1 / 2$ in (47).

\subsection{Numerical results}

We now present some numerical experiments on the approximation of solutions to the backward resolution. We take again system (45) recalled below

$$
\left\{\begin{array}{l}
u_{t}+\left(\frac{u^{2}}{2}\right)_{x}=0, \\
v_{t}+\left(u^{2} v\right)_{x}=0 .
\end{array}\right.
$$

Differently from Example 3, now we consider (48) in a non resonant situation. Therofore we set $(48)$ on the domain $[0.1,1] \times[-0.5,0.5]$. We consider as final state for the Burgers equation,

$$
u_{T}(x)=\left\{\begin{array}{l}
x+0.4 \text { if } x<0 \\
x-0.3 \text { if } x \geq 0
\end{array}\right.
$$

and we choose two types of final state for the continuity equation

$$
v_{T}^{(1)}(x)=\left\{\begin{array}{l}
0.75 \text { if } x<0, \\
-0.25 \text { if } x \geq 0
\end{array} \quad \text { and } v_{T}^{(2)}(x)=0.3 \sin (4 \pi x) .\right.
$$


Now choosing $\Delta x=1.56 \times 10^{-3}, \Delta t=\Delta x / 2$ and performing a backward resolution of (48) by using the numerical scheme presented in 5.1, we compute the approximate initial states denoted by $u_{\sigma}^{0}, v_{\sigma}^{(1)}$ and $v_{\sigma}^{(2)}$ respectively. We next check that solving (48) until time $T=1$, starting from $u_{\sigma}$ and $v_{\sigma}^{(1)}, v_{\sigma}^{(2)}$ respectively, we obtain final states denoted by $u^{N+1}, v^{(1)^{N+1}}$ and $v^{(2)^{N+1}}$ that are good approximations of $u_{T}$, $v_{T}^{(1)}$ and $v_{T}^{(2)}$ respectively. To that end, we define the following relative $L^{1}$-discrete error norms

$$
e_{b}=\frac{\sum_{j=1}^{M}\left|u_{T}\left(x_{j}\right)-u_{j}^{N+1}\right|}{\sum_{j=1}^{M}\left|u_{T}\left(x_{j}\right)\right|} \text { and } e_{t}^{(k)}=\frac{\sum_{j=1}^{M}\left|v_{T}^{(k)}\left(x_{j}\right)-v_{j}^{(k)^{N+1}}\right|}{\sum_{j=1}^{M}\left|v_{T}^{(k)}\left(x_{j}\right)\right|},
$$

where $k=1$ or 2 . We observe that the error norms $e_{b}, e_{t}^{(1)}$ and $e_{t}^{(2)}$ converge to zero, as reported in the following table:

\begin{tabular}{|c|c|c|c|}
\hline$\Delta x$ & $e_{b}$ & $e_{t}^{(1)}$ & $e_{t}^{(2)}$ \\
\hline 0.05 & 0.3 & 0.065 & 0.2 \\
\hline 0.025 & 0.23 & 0.041 & 0.12 \\
\hline 0.0125 & 0.16 & 0.023 & 0.073 \\
\hline $6.25 \times 10^{-3}$ & 0.1 & 0.019 & 0.044 \\
\hline $3.125 \times 10^{-3}$ & 0.068 & $7.2 \times 10^{-3}$ & 0.026 \\
\hline $1.56 \times 10^{-3}$ & 0.041 & $3.9 \times 10^{-4}$ & 0.015 \\
\hline $7.8 \times 10^{-4}$ & 0.023 & $2.2 \times 10^{-4}$ & $8.8 \times 10^{-3}$ \\
\hline
\end{tabular}

In addition, in Figure 1 we have plotted the exact and the final states with the approximate ones for $\Delta x=1.56 \times 10^{-3}$ and $\Delta t=\Delta x / 2$. We observe that apart from the numerical diffusion of the scheme, the final states are correctly computed. Note that the accuracy of the scheme can be improved by using hight order schemes (see for instance [29]).

We now turn to the backward resolution of the following resonant system that was already considered in Example 2:

$$
\left\{\begin{array}{l}
u_{t}+\left(\frac{u^{2}}{2}\right)_{x}=0, \\
v_{t}+(u v)_{x}=0 .
\end{array}\right.
$$

We keep the same domain, space step and time step as in the non resonant case. We still consider the final states (49)-(50). Proceeding as previously, starting from the computed initial states, we solve system (51) forward in time and we compare the exact and the computed final states. We report the result in Figure 2.

We notice that the computed final states do not approximate the exact final states as well as in the non resonant case. This observation is reinforced with the very slow convergence of the relative errors $e_{t}^{(1)}$ and $e_{t}^{(2)}$ in Table 1 . 

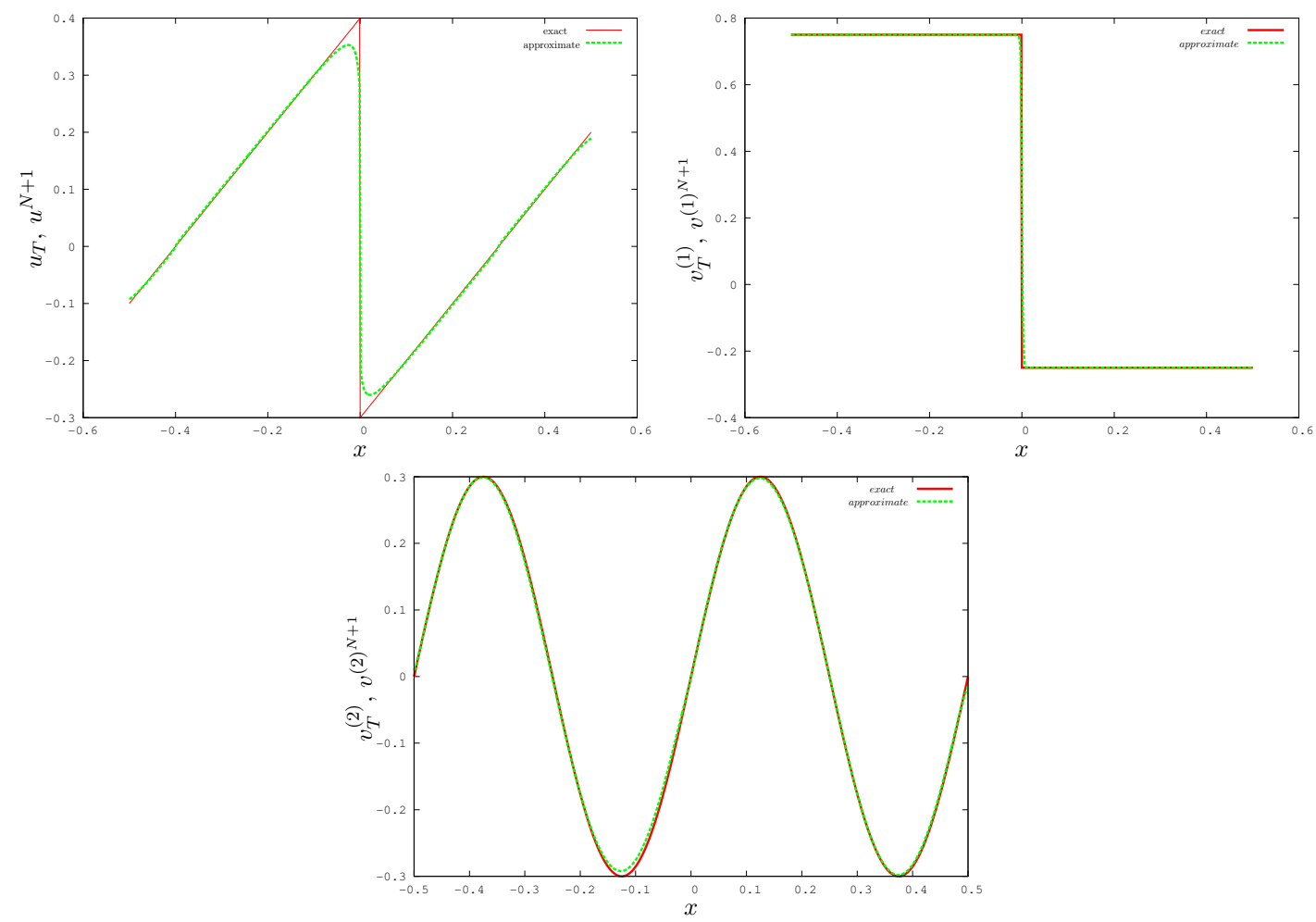

Figure 1: Non resonant case

In order to improve the accuracy of the approximation, we consider a final state $u_{T}$ that belongs to $\mathcal{A}_{T+\delta}$, for $\delta>0$. In practice, we first solve the Burgers equation on the time interval $[T, T+\delta]$ with $u_{T}$ as initial state. We next perform a backward resolution to compute $u_{\sigma}, v_{\sigma}^{(k)}, k=1$ or 2 . This procedure amounts to first regularize the Burgers' final state (using the procedure of the proof of Lemma 2.1) then perform the backward resolution.

Finally, as we have done before, we solve the problem forward in time. The results are reported in Figure 3 which shows the better approximation of the exact final states $v_{T}^{(1)}$ and $v_{T}^{(2)}$. The regularization procedure also allows to improve the rate of convergence of the $L^{1}$ error norms denoted by $e_{t, R}^{(k)}, \mathrm{k}=1,2$, in Table 1.

\section{Conclusions}

Our results concern attainability and backward resolution of systems of form (1). They include, after an appropriate reformulation, the classical Keyfitz-Kranzer and multi-component chromatography systems.

Assuming that system (1) admits nontrivial entropies, we characterize the entropies, describe the attainable set $\mathfrak{A}_{T}=\mathcal{A}_{T} \times L^{\infty}$ for (1) and for every $U_{T} \in \mathfrak{A}_{T}$ we prove the existence of a unique isentropic (and therefore, admissible) solution of system (1) satisfying $U(T)=U_{T}$. Moreover, we give a constructive algorithm that selects this isentropic solution. 

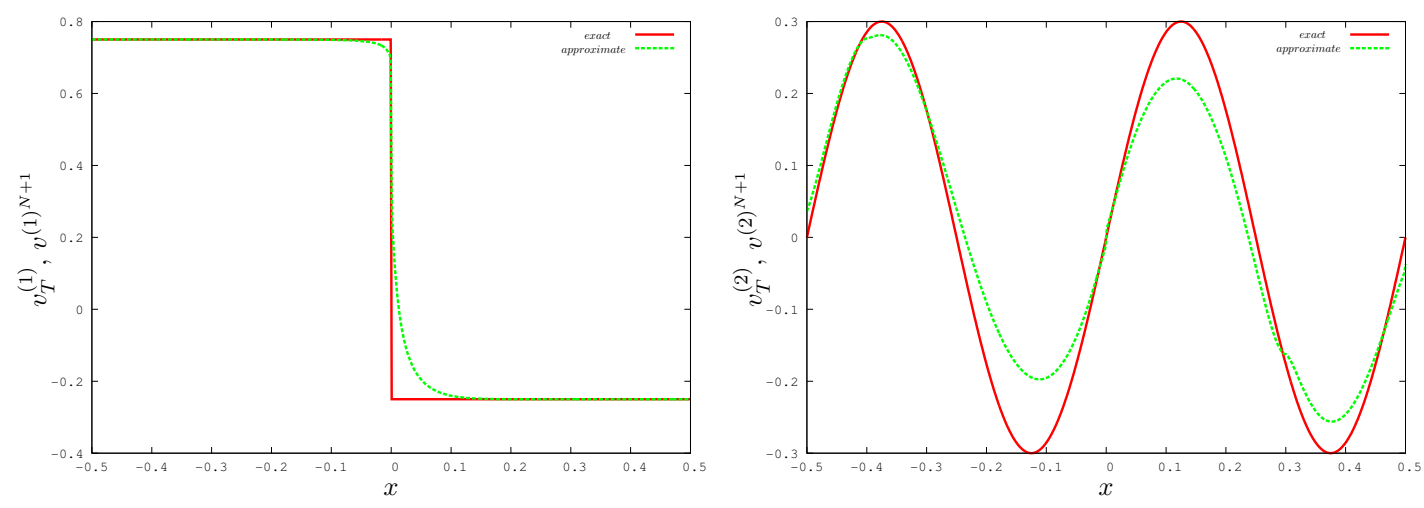

Figure 2: Resonant case

\begin{tabular}{|c|c|c|c|c|}
\hline$\Delta x$ & $e_{t}^{(1)}$ & $e_{t, R}^{(1)}$ & $e_{t}^{(2)}$ & $e_{t, R}^{(2)}$ \\
\hline 0.05 & 0.15 & 0.11 & 0.74 & 0.43 \\
\hline 0.025 & 0.11 & 0.081 & 0.65 & 0.3 \\
\hline 0.0125 & 0.086 & 0.037 & 0.57 & 0.19 \\
\hline $6.25 \times 10^{-3}$ & 0.058 & 0.015 & 0.48 & 0.1 \\
\hline $3.125 \times 10^{-3}$ & 0.038 & $8 \times 10^{-3}$ & 0.35 & 0.06 \\
\hline $1.56 \times 10^{-3}$ & 0.023 & $4.4 \times 10^{-3}$ & 0.21 & 0.032 \\
\hline $7.8 \times 10^{-4}$ & 0.014 & $3.1 \times 10^{-3}$ & 0.12 & 0.016 \\
\hline
\end{tabular}

Table 1: Convergence of the $L^{1}$-error norms

Further, when system (1) does not admit nontrivial entropies (this is the case for some resonant systems) we consider that the function $U=(u, v)$ is an admissible solution for the system if $u$ is an entropy solution for the scalar conservation law and the function $v$ is renormalized solution of the companion continuity equation in the sense of DiPerna and Lions. In this case we have approximate controllability for every $U_{T} \in \mathfrak{A}_{T}$, and exact controllability in a dense subset $\mathbb{A}_{T} \times L^{\infty}$ of $\mathfrak{A}_{T}$. We show that the same numerical algorithm for backward solution applies starting from any $U_{T} \in \mathfrak{A}_{T}$, but that its efficiency may drop. In this case, a simple regularization procedure inspired by the definition of $\mathbb{A}_{T}$ permits to improve the performance of the approximation scheme. This is also the case of the convergence of the relative $L^{1}$-error norms as reported Table 1.

\section{Appendix: backward construction for (6) revisited}

The algorithm we present below is less general than the original one in [1], in the sense that we do not allow for an arbitrary behavior of the limit function $u_{0}$ outside the interval $I=\left[\rho\left(C_{1}\right), \rho\left(C_{2}\right)\right]$. Roughly speaking, in [1], the initial condition in a neighborhood of $\rho\left(C_{1}\right)$ and $\rho\left(C_{2}\right)$ was selected in order to create sufficiently strong shocks able to neutralize all possible waves coming from outside of the interval $I$; note in passing that in this case, solutions cannot be isentropic. Here, we want to 

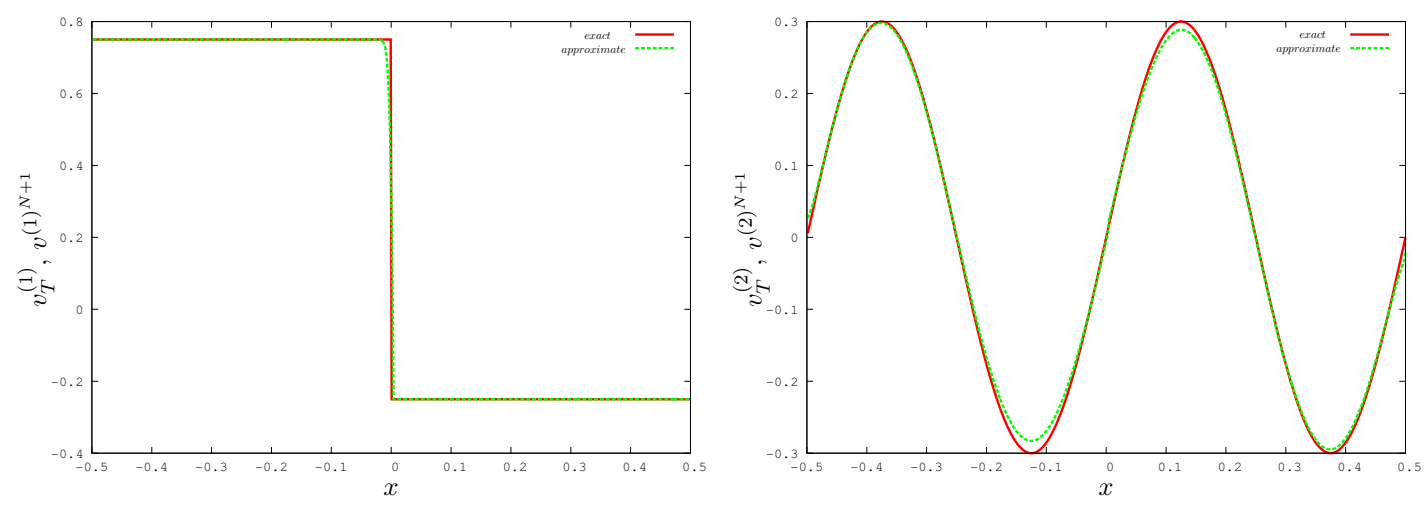

Figure 3: Resonant case with regularisation

handle the case in which the profile of the solution at time $T$ is fixed on $J=\mathbb{R}$; our result will be obtained by a cut-and-paste argument from the local construction, see Section 7.2. In return, we can bypass the hypotheses of uniform convexity and of superlinear growth of the flux function $f$ made in [1], as such assumptions were only used to prevent the possibly large data outside $I$ to interfere with the backward construction.

In the sequel, we fix $u_{T} \in \mathcal{A}_{T}(\mathbb{R}, f)$. It is clear that for every $I \subset \mathbb{R}$, the restriction $\left.u_{T}\right|_{I}$ belongs to $\mathcal{A}_{T}(I, R)$. First we present the backward construction on a bounded subset $I$ or $\mathbb{R}$, where we extend $u_{T} \mid I$ by constant values on the two connected components of $\mathbb{R} \backslash I$. Then we juxtapose the obtained solutions by using carefully chosen partitions $\cup_{k \in \mathbb{Z}} I_{k}=\mathbb{R}=\cup_{k \in \mathbb{Z}} J_{k}$ of the whole real line.

\subsection{The isentropic backward algorithm on a bounded domain}

- First notice that Lax-Oleinik formula can be applied to describe explicitly the solution of a scalar conservation law as soon as the flux function has a 1-to-1 continuous derivative. Thus we assume, to start with, that $f^{\prime}$ is strictly monotone and surjective; we indicate at the end of the paragraph the easy observations needed to treat the general case of merely injective continuous function $f^{\prime}$.

- We express $\rho$ from $u_{T} \in \mathcal{A}_{T}(I, f)$ by applying Lax-Oleinik formula, i.e. we set $\rho(x)=x-T f^{\prime}\left(u_{T}(x)\right)$ defined on the appropriate interval $J$ given by $I=\rho(J)$. Next, for any given partition of $I, y_{0}=A_{1}<\ldots<y_{n}=A_{2}$, we construct a partition of $J, x_{0}=C_{1} \leq \ldots \leq x_{n}=C_{2}$ as follows ${ }^{1}$. If the function $\rho$ were invertible we would just say that $x_{i}=\rho^{-1}\left(y_{i}\right)$. However, this is not the case when the target state $u_{T}$ contains a shock, therefore we define

$$
x_{i}=\sup \left\{x: \rho(x)<y_{i}\right\}
$$

and

$$
\rho_{n}(x)=\sum_{i=1}^{n-1} y_{i} \chi_{\left(x_{i}, x_{i+1}\right]}(x)+y_{0} \chi_{\left[x_{0}, x_{1}\right]}(x) .
$$

${ }^{1}$ The precise notation for the partitions of $I$ and $J$, respectively, would be $y_{0}^{n}<y_{1}^{n}<\cdots<y_{n}^{n}$ and $x_{0}^{n} \leq x_{1}^{n} \leq \cdots \leq x_{n}^{n}$; in the sequel, we drop the superscript $n$ for the sake of readability. 
Two important remarks are in order. First, some of the $x_{i}$ may coincide, see Fig. 4. Second, we can construct a piecewise affine approximation $u_{T, n}$ of $u_{T}$ by using the
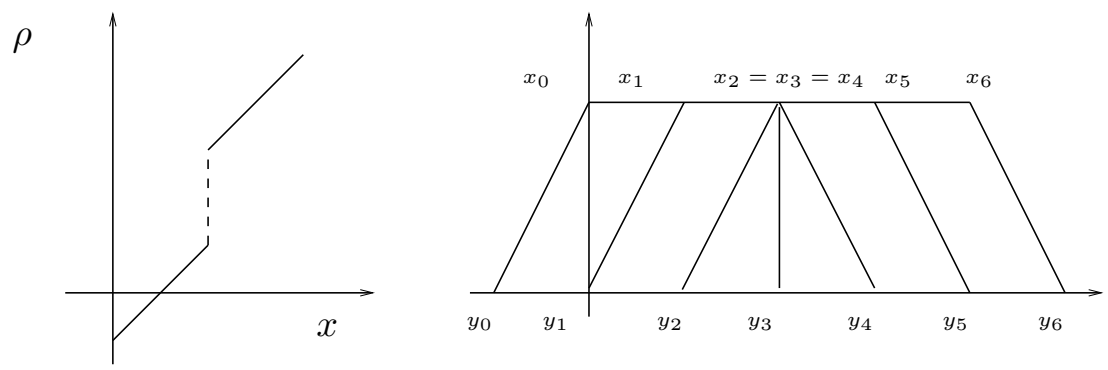

Figure 4: The function $\rho$ and the regions $\Omega_{i}$ associated to $u_{T}(x)=\operatorname{sign}(1-x)$.

Lax-Oleinik formula and the piecewise constant approximation $\rho_{n}$ of $\rho$. Notice that the functions $u_{T, n}$ are not constant when $u_{T}$ is constant and that, in general, their total variation is much larger that the total variation of $u_{T}$, see Fig. 5 . While



Figure 5: The profiles $u_{n}(t, \cdot)$ (with $n=4$ ) at different times associated to the target $u_{T} \equiv 1$, with $T=1 / 2$ and $f(u)=\frac{u^{2}}{2}$.

the construction up to this point works for any choice of the partition in $I$, we need to impose the condition $\left|y_{i}-y_{i+1}\right| \leq \frac{K}{n}$, for some positive constant $K$, in order to achieve uniform convergence $\rho_{n}(x) \rightarrow \rho(x)$. The pointwise convergence of the sequence $\rho_{n}$ implies the pointwise convergence of $u_{T, n}$ to $u_{T}$, by virtue of the Lax-Oleinik formula. 


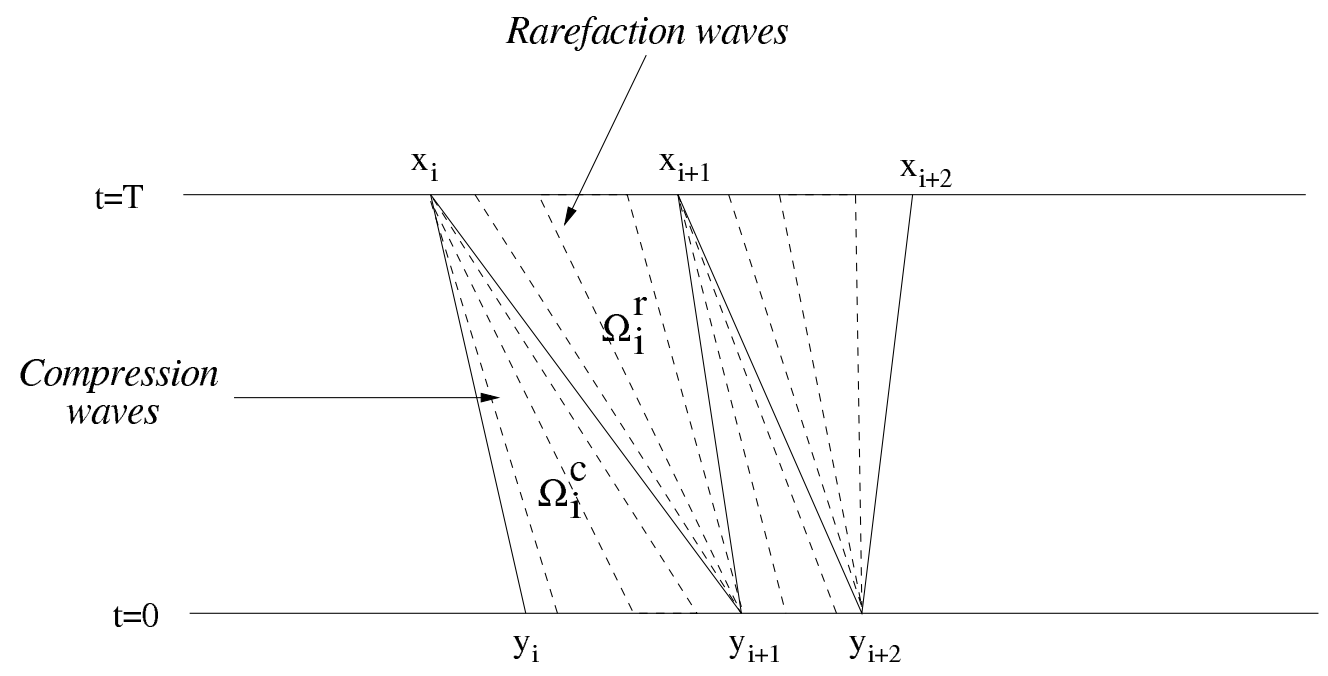

Figure 6: The regions $\Omega_{i}$.

- We call $l(t, A, C)$ the line in the $x-t$ plane joining the points $(0, A)$ and $(T, C)$. We trace the lines $l\left(t, y_{i}, x_{i}\right)$ and $l\left(t, y_{i+1}, x_{i}\right)$, for $i=0, \ldots n-1$, plus the line $l\left(t, y_{n}, x_{n}\right)$. Of course, some of these lines may coincide. We call $\Omega_{i}$ the region bounded by the line segments $l\left(t, y_{i}, x_{i}\right), l\left(t, y_{i+1}, x_{i+1}\right), t=0$ and $t=T$.

- We construct the solution $u_{n}$ in each of the regions $\Omega_{i}, i=0, \ldots, n-1$. For the reader's convenience we will denote by $\Omega_{i}^{c}$ the triangular region bounded by the line segments $l\left(t, y_{i}, x_{i}\right), l\left(t, y_{i+1}, x_{i}\right)$ and $t=0$, and by $\Omega_{i}^{r}$ the region $\Omega_{i} \backslash \Omega_{i}^{c}$, see figure 6 .

We have to consider two cases : $x_{i} \neq x_{i+1}$ and $x_{i}=x_{i+1}$. In the first case $u_{n}$ consists of a compression wave in $\Omega_{i}^{c}$ and a rarefaction wave in $\Omega_{i}^{r}$

$$
u_{n}(t, x)= \begin{cases}\left(f^{\prime}\right)^{-1}\left(\frac{x-x_{i}}{t-T}\right), & \text { for }(t, x) \in \Omega_{i}^{c}, \\ \left(f^{\prime}\right)^{-1}\left(\frac{x-y_{i+1}}{t}\right), & \text { for }(t, x) \in \Omega_{i}^{r} .\end{cases}
$$

In the second case the region $\Omega_{i}^{r}$ reduces to a line, because the lines $l\left(t, y_{i+1}, x_{i}\right)$ and $l\left(t, y_{i+1}, x_{i+1}\right)$ coincide. Then we have

$$
u_{n}(t, x)=\left(f^{\prime}\right)^{-1}\left(\frac{x-x_{i}}{t-T}\right), \quad \text { for }(t, x) \in \Omega_{i} .
$$

The function $u_{n}$ is set to be constant on the unbounded strips ] $-\infty, l\left(t, A_{1}, C_{1}\right)$ ] and $\left[l\left(t, A_{2}, C_{2}\right),+\infty\left[\right.\right.$, with values $u_{T}\left(C_{1}\right)$ and $u_{T}\left(C_{2}\right)$ respectively.

- The function $u_{n}$ extends to the lines $t=0$ and $t=T$ so that $u_{n} \in C(] 0, T\left[; L_{l o c}^{1}\right)$, and $\lim _{t \rightarrow T} u_{n}(t, x)=u_{T, n}(x)$. The explicit form for the initial condition $u_{0, n}$ corresponding to the approximate target profile $u_{T, n}$ is

$$
\begin{aligned}
u_{0, n}(x)= & \sum_{i=1}^{n-2}\left(f^{\prime}\right)^{-1}\left(\frac{x_{i}-x}{T}\right) \chi_{\left(y_{i}, y_{i+1}\right]} \\
& +\left(f^{\prime}\right)^{-1}\left(\frac{x_{0}-x}{T}\right) \chi_{\left(-\infty, y_{1}\right]}+\left(f^{\prime}\right)^{-1}\left(\frac{x_{n-1}-x}{T}\right) \chi_{\left(y_{n-1},+\infty\right]} .
\end{aligned}
$$


The so constructed function is a continuous, piecewise regular solution of (6) consisting of alternating compression and rarefaction waves as shown in Fig. 6. It is easily checked that $u_{n}$ is in fact an isentropic solution of $(6)$ on $[0, T] \times \mathbb{R}$ (e.g., one can apply the general result of Korobkov and Panov [27]).

- We aim to apply Helly's theorem, see for exemple [10], to prove the $L_{l o c}^{1}$ convergence for the sequence $f^{\prime}\left(u_{0, n}\right)$. We notice that

$$
\begin{aligned}
& T V\left(f^{\prime}\left(u_{n}(t, \cdot)\right)\right)=\sum_{i=0}^{n-1}\left|f^{\prime}\left(u_{n}\left(t, l\left(t, y_{i}, x_{i}\right)\right)\right)-f^{\prime}\left(u_{n}\left(t, l\left(t, y_{i+1}, x_{i}\right)\right)\right)\right| \\
& +\sum_{i=1}^{n-1}\left|f^{\prime}\left(u_{n}\left(t, l\left(t, y_{i+1}, x_{i}\right)\right)\right)-f^{\prime}\left(u_{n}\left(t, l\left(t, y_{i+1}, x_{i+1}\right)\right)\right)\right| \\
& =\sum_{i=0}^{n-1}\left|\frac{x_{i}-y_{i}}{T}-\frac{x_{i}-y_{i+1}}{T}\right|+\sum_{i=1}^{n-1}\left|\frac{x_{i}-y_{i+1}}{T}-\frac{x_{i+1}-y_{i+1}}{T}\right| \\
& =\frac{\left|A_{2}-A_{1}\right|+\left|C_{2}-C_{1}\right|}{T} .
\end{aligned}
$$

Moreover, $\left|f^{\prime}\left(u_{n}(t, x)\right)\right|$ is bounded by the constant

$$
M=\frac{\max \left(C_{2}, A_{2}\right)-\min \left(C_{1}, A_{1}\right)}{T} \quad \forall(t, x) \in[0, T] \times \mathbb{R} .
$$

Observe that all the above estimates are valid up to $t=T$ and $t=0$ included, therefore the sequence $f^{\prime}\left(u_{0, n}\right)$ converges a.e. in $L_{l o c}^{1}(I)$ to a function $F$. By the continuity of $\left(f^{\prime}\right)^{-1}$ we can define $u_{0}=\left(f^{\prime}\right)^{-1}(F)$ and deduce that there exists a subsequence of $u_{0, n}$ converging a.e. to $u_{0}$.

- Call $u$ the unique entropy solution of the conservation law in $[0, T] \times \mathbb{R}$ corresponding to the initial condition $u_{0}$. Using the $L^{1}$-contraction property we readily show that for all $t \in(0, T]$ the sequence $u_{n}(t)$ also converges to $u(t)$, and that $\left.u(T)\right|_{I}$ coincide with $u_{T} \mid I$ a.e.

Because each $u_{n}$ is an isentropic solution of $(6)$ in $[0, T] \times \mathbb{R}$, it follows that $u_{n}$ is also an isentropic solution. Indeed, the set of isentropic solutions is closed under the $L_{l o c}^{1}$ convergence.

- Finally, notice that whenever $f^{\prime}$ is strictly increasing but not surjective, and a target $u_{T}$ is fixed, we can modify $f$ outside the interval $\left[-\left\|u_{T}\right\|_{\infty},\left\|u_{T}\right\|_{\infty}\right]$

\subsection{Target state given on an unbounded domain}

In this section we apply the construction presented in Section 7.1 to solve backwards the nonlinear scalar conservation law

$$
u_{t}+f(u)_{x}=0,
$$

with fixed target state $u_{T} \in L^{\infty}(\mathbb{R})$ in the admissible set $\mathcal{A}_{T}(\mathbb{R}, f)$. We start by noticing that, since $u_{T}$ belongs to $L^{\infty}(\mathbb{R})$, the associated function $\rho$ is continuous and strictly monotone outside a set of bounded measure. Then it is possible to find a sequence of points $A_{k}, k \in \mathbb{N}$, such that

1. $\mathbb{R}=\cup_{k}\left[A_{k}, A_{k+1}[\right.$, 
2. the inverse function $\rho^{-1}$ is well defined on a neighborhood of $A_{k}$. We call $C_{k}$ the point $\rho^{-1}\left(A_{k}\right)$.

We stress that the second condition above means in particular that $u_{T}$ is continuous at all points $C_{k}$.

In order to construct the backward solution $u$ and the initial condition $u_{0}$, we implement the algorithm defined in the previous section with $I=\left[A_{k}, A_{k+1}\right], J=$ $\left[C_{k}, C_{k+1}\right]$, and

$$
u_{T, k}= \begin{cases}u_{T}\left(C_{k}\right), & \text { for } x \leq C_{k}, \\ u_{T}(x), & \text { for } x \in] C_{k}, C_{k+1}[, \\ u_{T}\left(C_{k+1}\right), & \text { for } x \geq C_{k+1} .\end{cases}
$$

This procedure gives us a solution $u_{k}$ which is constant on each side from the interval $\left[l\left(t, A_{k}, C_{k}\right), l\left(t, A_{k+1}, C_{k+1}\right)\right]$, for all $\left.t \in\right] 0, T[$. Analogously the initial condition $u_{0, k}$ is constant outside $\left[A_{k}, A_{k+1}\right]$. Clearly, the values taken by $u_{k}$ and $u_{0, k}$ for $x \leq l\left(t, A_{k}, C_{k}\right)$ and $x \geq l\left(t, A_{k+1}, C_{k+1}\right)$ are $u_{T}\left(C_{k}\right)$ and $u_{T}\left(C_{k+1}\right)$ respectively. The solution $u$ and the initial condition $u_{0}$ are obtained by piecing together the $\left(u_{k}\right)$ 's and the $\left(u_{0, k}\right)$ 's as follows:

$$
\begin{aligned}
& u(t, x)=\sum_{k} u_{k}(t, x) \chi_{\left[l\left(t, A_{k}, C_{k}\right), l\left(t, A_{k+1}, C_{k+1}\right)[\right.} \\
& u_{0}(x)=\sum_{k} u_{0, k}(x) \chi_{\left[A_{k}, A_{k+1}[\cdot\right.}
\end{aligned}
$$

The only possible problem in this construction, namely the fact that one may actually introduce jumps discontinuities in $u$ and $u_{0}$ in by putting side by side two different values, is ruled out by our choice of the sequence $\left(A_{k}\right)_{k}$.

\section{References}

[1] Adimurthi, S. S. Ghoshal, and G. D. Veerappa Gowda. Exact controllability of scalar conservation law with strict convex flux. To appear in Mathematical Control and Related Fields, 2014.

[2] Adimurthi, S.S. Ghoshal, and G. D. Veerappa Gowda. Optimal controllability for scalar conservation laws with strict convex flux. preprint, 2011.

[3] L. Ambrosio. Transport equation and Cauchy problem for $B V$ vector fields. Invent. Math., 158(2):227-260, 2004.

[4] L. Ambrosio, F. Bouchut, and C. De Lellis. Well-posedness for a class of hyperbolic systems of conservation laws in several space dimensions. Comm. Partial Differential Equations, 29(9-10):1635-1651, 2004.

[5] L. Ambrosio, G. Crippa, A. Figalli, and L. V. Spinolo. Some new well-posedness results for continuity and transport equations, and applications to the chromatography system. SIAM J. Math. Anal., 41(5):1890-1920, 2009.

[6] L. Ambrosio and C. De Lellis. Existence of solutions for a class of hyperbolic systems of conservation laws in several space dimensions. Int. Math. Res. Not., (41):2205-2220, 2003 
[7] F. Ancona and A. Marson. On the attainable set for scalar nonlinear conservation laws with boundary control. SIAM J. Control Optim., 36(1):290-312 (electronic), 1998.

[8] F. Bouchut and F. James. Équations de transport unidimensionnelles à coefficients discontinus. C. R. Acad. Sci. Paris Sér. I Math., 320(9):1097-1102, 1995.

[9] F. Bouchut and F. James. One-dimensional transport equations with discontinuous coefficients. Nonlinear Anal., 32(7):891-933, 1998.

[10] A. Bressan. Hyperbolic systems of conservation laws, volume 20 of Oxford Lecture Series in Mathematics and its Applications. Oxford University Press, Oxford, 2000. The one-dimensional Cauchy problem.

[11] J.-M. Coron. Global asymptotic stabilization for controllable systems without drift. Math. Control Signals Systems, 5(3):295-312, 1992.

[12] G. Crippa and L. V. Spinolo. An overview on some results concerning the transport equation and its applications to conservation laws. Commun. Pure Appl. Anal., 9(5):1283-1293, 2010.

[13] C. M. Dafermos. Generalized characteristics and the structure of solutions of hyperbolic conservation laws. Indiana Univ. Math. J., 26(6):1097-1119, 1977.

[14] C. M. Dafermos. Hyperbolic conservation laws in continuum physics, volume 325 of Grundlehren der Mathematischen Wissenschaften [Fundamental Principles of Mathematical Sciences]. Springer-Verlag, Berlin, third edition, 2010.

[15] C. De Lellis. Notes on hyperbolic systems of conservation laws and transport equations. In Handbook of differential equations: evolutionary equations. Vol. III, Handb. Differ. Equ., pages 277-382. Elsevier/North-Holland, Amsterdam, 2007.

[16] R. J. DiPerna and P.-L. Lions. Ordinary differential equations, transport theory and Sobolev spaces. Invent. Math., 98(3):511-547, 1989.

[17] E. Fatemi, B. Engquist, and S. Osher. Numerical solution of the high frequency asymptotic expansion for the scalar wave equation. J. Comput. Phys., 120(1):145-155, 1995.

[18] H. Freistühler. On the Cauchy problem for a class of hyperbolic systems of conservation laws. J. Differential Equations, 112(1):170-178, 1994.

[19] H. Frid. Asymptotic stability of non-planar Riemann solutions for multi-D systems of conservation laws with symmetric nonlinearities. J. Hyperbolic Differ. Equ., 1(3):567-579, 2004.

[20] S. S. Ghoshal. Finer analysis of characteristic curve and its application to exact, optimal controllability, structure of the entropy solution of a scalar conservation law with convex flux. PhD Thesis. TIFR CAM, Bangalore, 2012.

[21] O. Glass and S. Guerrero. On the uniform controllability of the Burgers equation. SIAM J. Control Optim., 46(4):1211-1238, 2007.

[22] E. Godlewski and P.-A. Raviart. Numerical approximation of hyperbolic systems of conservation laws, volume 118 of Applied Mathematical Sciences. SpringerVerlag, New York, 1996. 
[23] L. Gosse and F. James. Numerical approximations of one-dimensional linear conservation equations with discontinuous coefficients. Math. Comp., 69(231):987-1015, 2000.

[24] S. Guerrero and O. Yu. Imanuvilov. Remarks on global controllability for the Burgers equation with two control forces. Ann. Inst. H. Poincaré Anal. Non Linéaire, 24(6):897-906, 2007.

[25] T. Horsin. On the controllability of the Burgers equation. ESAIM Control Optim. Calc. Var., 3:83-95 (electronic), 1998.

[26] B. L. Keyfitz and H. C. Kranzer. A system of nonstrictly hyperbolic conservation laws arising in elasticity theory. Arch. Rational Mech. Anal., 72(3):219-241, $1979 / 80$.

[27] M. V. Korobkov and E. Yu. Panov. On isentropic solutions of first-order quasilinear equations. Mat. Sb., 197(5):99-124, 2006.

[28] M. Léautaud. Uniform controllability of scalar conservation laws in the vanishing viscosity limit. SIAM J. Control and Optimization, 50(3):1661-1699, 2012.

[29] R. J. LeVeque. Finite volume methods for hyperbolic problems. Cambridge Texts in Applied Mathematics. Cambridge University Press, Cambridge, 2002.

[30] E. Yu. Panov. On the theory of entropy solutions of the Cauchy problem for a class of nonstrictly hyperbolic systems of conservation laws. Mat. Sb., 191(1):127-157, 2000.

[31] E. Yu. Panov. Generalized solutions of the Cauchy problem for a transport equation with discontinuous coefficients. In Instability in models connected with fluid flows. II, volume 7 of Int. Math. Ser. (N. Y.), pages 23-84. Springer, New York, 2008.

[32] D. Serre. Systems of conservation laws. I. Cambridge University Press, Cambridge, 1999. Hyperbolicity, entropies, shock waves.

[33] J. Smoller. Shock waves and reaction-diffusion equations, volume 258 of Grundlehren der Mathematischen Wissenschaften [Fundamental Principles of Mathematical Sciences]. Springer-Verlag, New York, second edition, 1994. 